\title{
Resolving the complex structure of the dust torus in the active nucleus of the Circinus galaxy ${ }^{\star} \star \star \star$
}

\author{
K. R. W. Tristram ${ }^{1}$, K. Meisenheimer ${ }^{1}$, W. Jaffe ${ }^{2}$, M. Schartmann ${ }^{1}$, H.-W. Rix ${ }^{1}$, Ch. Leinert ${ }^{1}$, S. Morel $^{3}$, \\ M. Wittkowski ${ }^{4}$, H. Röttgering ${ }^{2}$, G. Perrin ${ }^{5}$, B. Lopez ${ }^{6}$, D. Raban ${ }^{2}$, W. D. Cotton ${ }^{7}$, U. Graser ${ }^{1}$, \\ F. Paresce ${ }^{4}$, and Th. Henning ${ }^{1}$
}

1 Max-Planck-Institut für Astronomie, Königstuhl 17, 69117 Heidelberg, Germany e-mail: [tristram;meise] @mpia.de

2 Leiden Observatory, Leiden University, Niels-Bohr-Weg 2, 2300 CA Leiden, The Netherlands

European Southern Observatory, Casilla 19001, Santiago 19, Chile

${ }^{4}$ European Southern Observatory, Karl-Schwarzschild-Strasse 2, 85748 Garching bei München, Germany

5 LESIA, UMR 8109, Observatoire de Paris-Meudon, 5 place Jules Janssen, 92195 Meudon Cedex, France

${ }^{6}$ Laboratoire Gemini, UMR 6203, Observatoire de la Côte d'Azur, BP 4229, 06304 Nice Cedex 4, France

7 NRAO, 520 Edgemont Road, Charlottesville, VA 22903-2475, USA

Received 27 July 2007 / Accepted 27 August 2007

\section{ABSTRACT}

\begin{abstract}
Aims. To test the dust torus model for active galactic nuclei directly, we study the extent and morphology of the nuclear dust distribution in the Circinus galaxy using high resolution interferometric observations in the mid-infrared.

Methods. Observations were obtained with the MIDI instrument at the Very Large Telescope Interferometer. The 21 visibility points recorded are dispersed with a spectral resolution of $\lambda / \delta \lambda \approx 30$ in the wavelength range from 8 to $13 \mu \mathrm{m}$. To interpret the data we used a stepwise approach of modelling with increasing complexity. The final model consists of two black body Gaussian distributions with dust extinction.

Results. We find that the dust distribution in the nucleus of Circinus can be explained by two components, a dense and warm disk-like component of $0.4 \mathrm{pc}$ size and a slightly cooler, geometrically thick torus component with a size of $2.0 \mathrm{pc}$. The disk component is oriented perpendicular to the ionisation cone and outflow and seems to show the silicate feature at $10 \mu \mathrm{m}$ in emission. It coincides with a nuclear maser disk in orientation and size. From the energy needed to heat the dust, we infer a luminosity of the accretion disk of $L_{\text {acc }}=10^{10} L_{\odot}$, which corresponds to $20 \%$ of the Eddington luminosity of the nuclear black hole. We find that the interferometric data are inconsistent with a simple, smooth and axisymmetric dust emission. The irregular behaviour of the visibilities and the shallow decrease of the dust temperature with radius provide strong evidence for a clumpy or filamentary dust structure. We see no evidence for dust reprocessing, as the silicate absorption profile is consistent with that of standard galactic dust. We argue that the collimation of the ionising radiation must originate in the geometrically thick torus component.

Conclusions. Based on a great leap forward in the quality and quantity of interferometric data, our findings confirm the presence of a geometrically thick, torus-like dust distribution in the nucleus of Circinus, as required in unified schemes of Seyfert galaxies. Several aspects of our data require that this torus is irregular, or "clumpy".
\end{abstract}

Key words. galaxies: active - galaxies: nuclei - galaxies: Seyfert - galaxies: individual: Seyfert - radiation mechanisms: thermal techniques: interferometric

\section{Introduction}

In the widely accepted model for low and intermediate luminosity active galactic nuclei (AGN), the central engine, consisting of a hot accretion disk around a supermassive black hole, and the broad line region (BLR) are surrounded by a torus of obscuring dust. Where the radiation from the central engine can escape, an ionisation cone and the narrow line region (NLR) are formed. Although motivated by the observation of broad emission lines in polarised light in type 2 AGN (torus edge-on), most tests of

\footnotetext{
* Based on observations collected at the European Southern Observatory, Chile, program numbers 073.A-9002(A), 060.A-9224(A), 074.B-0213(A/B), 075.B-0215(A) and 077.B-0026(A).

$\star \star$ Table 1 and Appendices A-C are only available in electronic form at http://www . aanda.org
}

this "unified model" rely on the shape of the overall spectral energy distribution (SED) and statistics, i.e. the number of objects where the central source is obscured or visible. Until recently, a direct spatially resolved assessment of the dust distribution that allegedly is responsible for the anisotropic absorption and obscuration has been missing. It is too small to be significantly resolved with single dish telescopes in the infrared, even for $10 \mathrm{~m}$ class telescopes at the diffraction limit. It has hence been impossible to directly confirm or disprove the existence and geometry of this essential component. Properties of the dust distribution, such as its size, temperature, geometry, alignment, and filling factor have hence remained unknown. A new approach is possible by studying the nearest AGN using interferometry at infrared wavelengths. The first objects to be successfully analysed in this way are the Seyfert 1 galaxy NGC 4151 (Swain et al. 2003) and 
the Seyfert 2 galaxy NGC 1068 (Wittkowski et al. 2004; Jaffe et al. 2004; Poncelet et al. 2006). In NGC 1068, which is the brightest galaxy in the mid-infrared (MIR), indeed an extended dust structure was resolved. In this paper we study the second brightest AGN in the MIR, the Circinus galaxy.

The Circinus galaxy is a highly inclined $\left(\sim 65^{\circ}\right) \mathrm{SA}(\mathrm{s}) \mathrm{b}$ galaxy harbouring a Seyfert type 2 active nucleus as well as a nuclear starburst. At about $4 \mathrm{Mpc}$ distance $(1 \operatorname{arcsec} \sim 20 \mathrm{pc}$, Freeman et al. 1977), the galaxy is among the nearest AGN and hence it is an ideal object to study the nuclear region of active galaxies. The nucleus is heavily obscured by dust lanes in the plane of the galaxy, so that it is best observed in the infrared. The galaxy can be considered a prototype Seyfert 2 object due to the presence of narrow emission lines (Oliva et al. 1994), broad emission lines in polarised light (Oliva et al. 1998), an ionisation cone traced by [O III], $\mathrm{H} \alpha$ and [Si VII] (Veilleux \& Bland-Hawthorn 1997; Maiolino et al. 2000; Wilson et al. 2000; Prieto et al. 2004), an outflow observed in CO (Curran et al. 1998, 1999), bipolar radio lobes (Elmouttie et al. 1998) and a Compton reflection component in X-rays (Matt et al. 1996; Smith \& Wilson 2001). The ionisation cone, the outflow and the radio lobes have a position angle of about $-40^{\circ}$, which is roughly perpendicular to the disk of the galaxy, to the circum-nuclear star forming rings and to the nuclear rings of molecular gas. Greenhill et al. (2003) have found an edgeon, warped "accretion" disk with a radius of $r_{\text {outer }} \sim 0.40 \mathrm{pc}$ which is traced by $\mathrm{H}_{2} \mathrm{O}$ masers. The almost Keplerian velocity curve yields an estimate of the mass of the central black hole to $M_{\mathrm{BH}}<(1.7 \pm 0.3) \times 10^{6} M_{\odot}$.

Modelling the SED of the nucleus of the Circinus galaxy was attempted by Siebenmorgen et al. (1997), Ruiz et al. (2001) and Schartmann et al. (2005). The latter two came to the conclusion that the presumed toroidal dust distribution is relatively small, with an effective emission region in the MIR of less than $3 \mathrm{pc}$ (i.e. $<0.15$ arcsec).

A detailed observational analysis in the mid-infrared was performed by Packham et al. (2005). The authors found extended emission with 2 arcsec size at position angles of $81^{\circ}$ and $278^{\circ}$, which is consistent with the edges of the aforementioned ionisation cones. However, their point spread function (PSF) analysis indicated that any extended nuclear emission in the midinfrared has sizes less than $0.20 \operatorname{arcsec}(4 \mathrm{pc})$ or is very weak. This showed again that single dish observations with current telescopes are not capable of resolving the nuclear dust distribution even for the nearest AGN, such as Circinus. For this reason, interferometric observations of the Circinus nucleus in the midinfrared with the Very Large Telescope Interferometer (VLTI) were performed.

This paper is organised as follows. In Sect. 2 we present the details of the observations and of the data reduction. Section 3 describes the properties of the interferometric data and the results directly deducible from them. Section 4 treats the modelling of the data. The interpretation of our findings and implications thereof are discussed in Sect. 5. Our conclusions are given in Sect. 6.

\section{Observations and data reduction}

\subsection{Instrument}

We obtained interferometric observations with the MID-infrared Interferometric instrument (MIDI) at the VLTI located on Cerro Paranal in northern Chile and operated by the European Southern Observatory (ESO). MIDI is a two beam Michelson type interferometer producing dispersed fringes in the $N$ band in a wavelength range from 8 to $13 \mu \mathrm{m}$ (Leinert et al. 2003). For our observations of Circinus, we combined the light of two of the $8.2 \mathrm{~m}$ unit telescopes (UTs) at any one time and used the low spectral resolution mode $(\lambda / \delta \lambda \approx 30)$ by insertion of a $\mathrm{NaCl}$ prism into the light path after the beam combiner.

\subsection{Observations}

The data were acquired between February 2004 and May 2006 during a total of six epochs. The first data set was obtained in February 2004 in science demonstration time (SDT). All following observations were carried out using guaranteed time observations (GTO). Table 1 gives a summary of the observations.

The observing sequence repeated for the science target and calibrator star consists of the following steps:

First, acquisition images of the two beams A and B coming from the unit telescopes are taken simultaneously. For this purpose, the standard chopping technique is used to suppress the background in the mid-infrared. For our observations, we used the default values for the chopping frequency ( $f=2 \mathrm{~Hz}$ ), chopping angle $(\alpha=0)$ and chopping throw $(\delta=15$ arcsec $)$ except for the observations on 2004 Jun. 03, where a smaller throw of $\delta=10 \operatorname{arcsec}$ was used. For the acquisition of Circinus, we used the $\mathrm{SiC}$ filter $(11.8 \pm 2.3 \mu \mathrm{m})$ at longer wavelengths because of the rising spectrum of the source. For the acquisition of the calibrator stars, which are in the Rayleigh-Jeans part of their spectra, we used the N8.7 filter $(8.6 \pm 1.5 \mu \mathrm{m})$ at shorter wavelengths. The acquisition procedure was repeated until the overlap of the two beams was within one pixel accuracy, that is within $\sim 86$ mas.

Then the beam combiner, the 0.6 arcsec wide slit and the $\mathrm{NaCl}$ prism are inserted into the light path leading to two spectrally dispersed interferograms of opposite phase. No chopping is needed during the interferometric measurement as the background is uncorrelated and cancels out in the data reduction process. The fringe signal is searched by scanning the optical path difference (OPD) over a few $\mathrm{mm}$ at the expected position. This is achieved by adjusting the VLTI delay lines. At the same time, a piezo-driven mirror in MIDI performs a sawtooth scanning pattern with a scan length of $80 \mu \mathrm{m}$ for one of the delay lines. This modulation generates moving fringes from which the optical path difference can be estimated. Once the zero optical path difference (0 OPD) is found, the so-called fringe track is started, during which the path difference is stabilised using the scanning of the piezo driven mirrors, while the VLTI delay lines compensate for siderial motion and atmospheric OPD shifts. There are two possibilities to track the fringes: the scans can pass over the white light fringe at 0 OPD or they are performed next to it. We preferred to observe in "offset tracking" mode with the OPD stabilised at $50 \mu \mathrm{m}$ from 0 OPD, because this is of advantage for a coherent data analysis (see below). The observations on 2004 Jun. 03 and 2005 Feb. 21 and the first visibility point on 2005 Mar. 01 were, however, observed tracking on the white light fringe. During these periods, the standard tracking software implemented at Paranal did not allow for using offset tracking.

Finally, photometric data are recorded using only one telescope at a time in the otherwise identical optical set-up. This results in two photometric data sets, one for each telescope. Again, chopping has to be used to suppress the background in the midinfrared.

Because our data were obtained over such a long period of time, the observation techniques evolved in the meantime. Additionally, the instrument itself has improved considerably 
since the first observations were made in February 2004. This leads to a large variety of exposure times ranging from DIT = $12 \mathrm{~ms}$ to $20 \mathrm{~ms}$. Similarly, the frame numbers range from NDIT = 5000 to 12000 for the fringe tracking and from $N D I T=1500$ to 4000 for the photometry (see Table 1 ). For the first 4 visibility points, only a tip tilt correction for the incoming wavefronts was possible using the STRAP units (Bonaccini et al. 2000). A much better correction was obtained with the MACAO adaptive optics system (Arsenault et al. 2003) since its implementation in late 2004, i.e. starting with our observations in February 2005. For Circinus, both systems were operated with a separate guide star at $50 \pm 2$ arcsec distance from the nucleus. For the observations of the calibrators, the wavefront sensing was performed using the stars themselves. The overall ambient conditions for all the observations were good to fair with seeing values from the site monitor varying from 0.5 to 1.8 arcsec. We had no problems with clouds or constraints due to wind, except for one observation. This observation ("sci05"), carried out in service mode on the UT2 - UT4 baseline, had to be aborted due to clouds building up over Paranal. Thus, no photometric data could be obtained for this measurement.

\subsection{Data reduction}

Not all measurements contain the full data set consisting of a fringe track and two photometric measurements, so that they could be reduced in a straightforward manner. This has three reasons: first of all, several measurements were performed at the end of the night or of the allocated time and only the fringe track could be obtained. Secondly, for some measurements the photometries proved to be unusable or to be of very low quality. Finally, for the measurement on the UT2 - UT4 baseline, no photometry could be obtained at all due to the adverse weather conditions. To nevertheless make use of all the fringe tracks obtained, we completed the fragmentary data sets with photometric data from the other measurements preferably using measurements as close as possible in time. The details are included in the last column of Table 1.

The data reduction was entirely performed by coherent visibility estimation using the software package EWS (Expert Work Station), written by Walter Jaffe. The alternative software package MIDI Interactive Analysis (MIA), which is based on a power spectrum analysis, was only used to cross-check the results for a selected number of observations: in these cases the two methods gave consistent results ${ }^{1}$. A detailed description of the coherent method can be found in Jaffe (2004). Here, we will concentrate only on the essential steps and highlight our specific configuration during the data reduction:

First, a fixed mask is applied to the two opposite phased interferograms, which are then collapsed to form two onedimensional interferograms. These are then subtracted with the result of doubling the signal while removing most of the background. As the fringes vary rapidly in time due to the scanning of the MIDI internal mirrors, a high-pass filter in temporal direction with a width of, in our case, 10 frames $($ smooth $=10)$ is applied to further remove the background. Additionally, the average of the entire spectrum is subtracted (dave $=1$ ), assuming a modulation of the fringe signal in frequency space. This is indeed the case, when the OPD is not zero, hence our preference to track next to the white light fringe. In the following two steps, the known instrumental OPD and the atmospheric

1 An online manual of both MIA and EWS is available at http://WwW.strw. leidenuniv.nl/ koehler/MIA+EWS-Manual/ delay are removed. The latter is estimated from the group delay $($ gsmooth $=10)$. Additionally a constant phase shift induced by the varying index of refraction of water vapour is estimated and also removed. After rejecting frames with too low or too high optical path difference, the remaining data is averaged coherently yielding the raw correlated flux, rawcor, and the differential phase, that is the relative variations of the phase with wavelength.

To determine the raw total flux of the target, first standard data reduction methods for chopped data are applied to the photometric data: the sky frames are subtracted from the object frames. As this does not lead to a satisfactory sky subtraction, two stripes on the detector frame, one above the target position and one below, are used to subtract an additional wavelength dependent sky estimate. Only for one data set an adjustment of the position of these stripes using the option dsky $=-2$ was necessary (see the respective comment in Table 1). The data are then compressed and averaged to a one-dimensional photometry, one for every telescope and side of the beam splitter ("channel"). Two sets of total fluxes are extracted: One is needed for the determination of the visibility, and hence the same mask as for the interferometric data is applied. To account for the possible inequality of the two beams from the two telescopes, this total flux is calculated as the geometric mean: rawtotg $=\sqrt{A_{1} \cdot B_{1}}+\sqrt{A_{2} \cdot B_{2}}$, with $A_{1}$ the photometry of beam $A$ (from the first telescope) in channel $1, B_{1}$ the photometry of beam $B$ (from the second telescope) in channel 1, as well as $A_{2}$ and $B_{2}$ the corresponding beams in channel 2 . The second total flux is determined without applying a mask and by taking the arithmetic mean of the individual measurements: rawtota $=\left(A_{1}+A_{2}+B_{1}+B_{2}\right) / 4$. The raw visibility is defined as rawvis = rawcor $/$ rawtotg. The same data reduction is applied to both the science target and to the calibrator star leading to the raw correlated flux scirawcor and calrawcor respectively, as well as the raw total fluxes scirawtotg, calrawtotg, scirawtota, calrawtota and the raw visibilities scirawvis and calrawvis.

Finally, the raw fluxes and visibilities of the science target are calibrated using the data of the calibrator star, to account for instrumental visibility losses and to determine the absolute flux values. For the calibration, we exclusively used the calibrator HD 120404 associated with Circinus, with the exception of one case, the long baseline UT2 - UT4, where HD 120404 was not observed at all. In this case, we used HD 107446 instead. The quality of the calibrators were checked by comparison to all the other calibrators observed during the same night. We decided to reject only one calibrator measurement because of its low correlated flux compared to the other measurements during the night: "cal14" observed on 2006 May 18 at 07:36:18. We corrected the Rayleigh-Jeans approximation for the template spectrum of the calibrator using a template spectrum, caltemplate, from a database provided by Roy van Boekel (private comm.). These template spectra were obtained by fitting stellar templates to multiband photometry.

To keep the influence of errors in the photometry as small as possible (especially considering the smaller number of usable photometries with respect to the number of successful fringe tracks), we calculated the calibrated correlated flux of Circinus according to $F_{\text {cor }}(\lambda)=$ scicalcor $=$ scirawcor/calrawcor $\cdot$ caltemplate using the option nophot $=1$ in EWS. The calibrated total flux is given by $F_{\text {tot }}(\lambda)=$ scicaltota $=$ scirawtota $/$ calrawtota $\cdot$ caltemplate and the calibrated visibility by $V(\lambda)=$ scicalvis = scirawvis $/$ calrawvis = (scirawcor/scirawtotg) - (calrawtotg/calrawcor). Note that in this case $V(\lambda) \neq F_{\text {cor }}(\lambda) / F_{\text {tot }}(\lambda)$. 


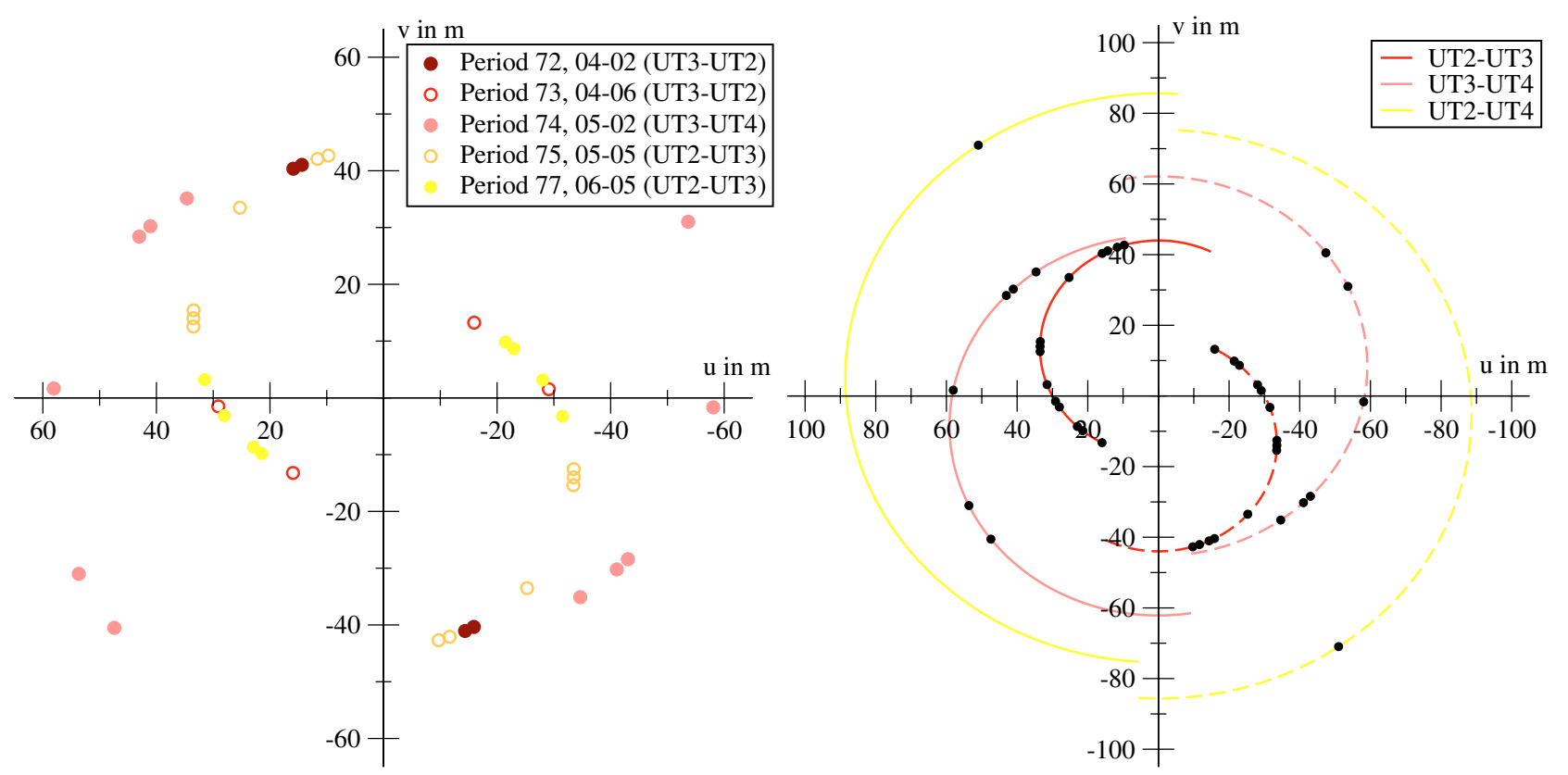

Fig. 1. Coverage of the $u v$ plane obtained for the Circinus galaxy with MIDI, the MID-infrared Interferometric instrument at the VLTI. The left panel shows the position of the observed fringe tracks in the $u v$ plane for the two shorter baselines, UT2 - UT3 and UT3 - UT4. The points are coded according to the epoch of observation. The right panel shows all the 21 observed fringe tracks as well as the $u v$ tracks of the baselines for the three combinations of unit telescopes that were used.

The entire data reduction and calibration was performed in the same manner for all visibility points without any further individual adjustment of the parameters, in order not to introduce any biases.

\section{4. uv coverage}

The interferometric data collected on Circinus constitute the most extensive interferometric data in the infrared or at shorter wavelengths of any extragalactic source to date. The coverage of the $u v$ plane achieved by all of the MIDI observations is shown in Fig. 1. Each point represents the position of a fringe track leading to a visibility point. Because we measure the interferometric signal of a real function (namely the brightness distribution) which has a symmetric Fourier transform, there are two points for each measurement, symmetrical to the centre. The different colours denote the different epochs of observation. We observed 21 fringe tracks and 31 usable photometries (note this is not the double of the fringe track number as it should optimally be; there are only 15 usable A photometries and 16 usable $B$ photometries) for Circinus as well as 12 fringe tracks plus 24 photometries for HD 120404, the calibrator star. From these data, we were able to reconstruct a total of 21 visibility points.

The distribution of the measurements in the $u v$ plane shown in Fig. 1 has concentrations at several locations, that is, two or three points at almost the same location. This was caused by consecutive measurements of the interferometric signal. The differences between such measurements can be considered as indications for the accuracy of the measurements themselves. Several measurements also share the same photometry or calibrator data. In a strict sense, the true number of absolutely independent measurements is thus only on the order of 12 , which is the number of independently measured calibrator data sets.

The observations were performed with only three baseline configurations: UT2 - UT3, UT2 - UT4 and UT3 - UT4. This causes the visibility points to lie on arcs in the $u v$ plane, which correspond to the classical $u v$ tracks known from radio interferometry. The $u v$ tracks for these three baseline configurations are plotted as continuous lines in the right panel of Fig. 1. The $u v$ coverage is far from optimal, as there are several regions lacking measurements: at a position angle of $-20^{\circ}$, no measurements were obtained at all and at a position angle of $+20^{\circ}$ only one baseline length $(B L=40 \mathrm{~m})$ was observed. The void regions therefore need to be filled in by future observations. In fact, the current coverage is unsuitable to attempt any image reconstruction as commonly done with radio interferometry data. There, a flux distribution can be directly determined from the data by an inverse Fourier transform and a prescription, such as the "clean algorithm". We tried applying such an algorithm to our data. However, the form of the PSF (the "beam") corresponding to our $u v$ coverage is very bad and shows strong periodic patterns, thereby dominating the outcome of the reconstruction process. The results of such an attempt are poor and hard to interpret, reflecting only general properties of the source. These general properties are much better constrained by models (see Sect. 4).

The differential phase (see Sect. 2.3) is less than $20^{\circ}$ for almost all baselines. At the shortest baselines, we find some evidence for a phase shift and there is also weak evidence for phase shifts at positions where the visibilities show unexpected behaviour (especially for sudden downturns at the edge of the spectal range covered). Nevertheless, we find no evidence for a major asymmetry in the source. We therefore postpone a detailed analysis of the differential phase information and concentrate in this paper on the interpretation of the visibility amplitude only.

\section{Results}

\subsection{Total flux}

Figure 2 shows the spectrum of the calibrated total flux, $F_{\text {tot }}(\lambda)$, of the Circinus nucleus. In several of the individual 


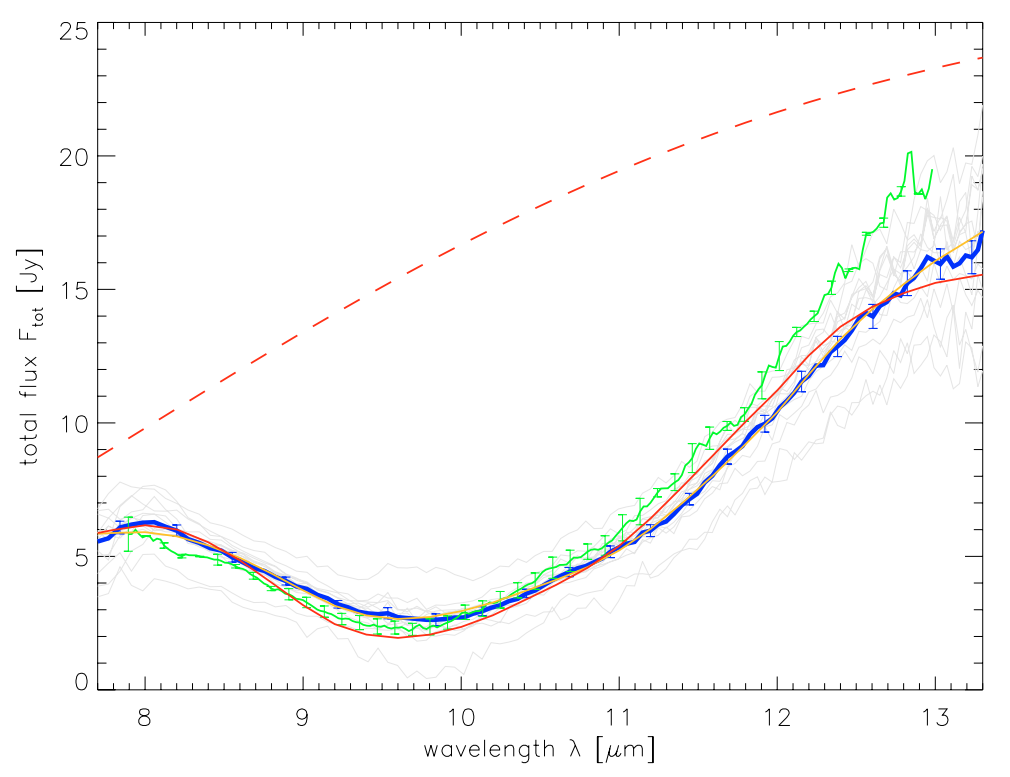

Fig. 2. Spectrum of the total flux of the Circinus nucleus in the $N$ band. The light grey curves are the 15 individual measurements of the total flux; error bars were omitted for the sake of clarity. The thick blue curve is the arithmetic mean of all the individual measurements, the error bars indicating the reduced standard deviation. The spectrum measured by Roche et al. (1991) is shown in green for comparison. The yellow curve shows the fit of the physical model to the total flux only (fit 1), while the red curve shows the fit to the full data set consisting of the total flux and the correlated fluxes (fit 2). The dashed red curve is the emission for the latter fit, without including the extinction by dust. For details on the modelling, see Sect. 4.2. measurements (light grey lines), enhanced noisiness is apparent between 9.5 and $10.0 \mu \mathrm{m}$, which is due to ozone absorption in the Earth's atmosphere. This is aggravated by the fact that the ozone feature coincides with the minimum of the flux in the intrinsic spectrum of Circinus. The individual measurements are all consistent with the average (thick, blue line), when the associated individual errors (not shown in Fig. 2) are considered. The curves with the largest deviations from the average come from measurements in 2004, which also have larger individual errors. The variations are mainly caused by imperfect background subtraction for early data or observations at high airmasses. We clearly see an increase in the data quality after the first measurements. The edges of the $N$ band are located at $8.2 \mu \mathrm{m}$ and $13.0 \mu \mathrm{m}$. Towards shorter and longer wavelengths, the atmosphere has a high opacity due to water absorption. Given the large number of measurements, the averaged total flux is nevertheless usable beyond these boundaries.

The broad absorption feature dominating most of the spectrum is due to silicate absorption. We see no evidence for any line emission or emission of Polycyclic Aromatic Hydrocarbons (PAH), as observed at larger distances from the nucleus (Roche et al. 2006) or in larger apertures. The nuclear spectrum measured by Roche et al. agrees with our measurement of the total flux within $20 \%$ (see Fig. 2). The differences are probably due to the higher spectral resolution $(R \sim 125)$ and the different orientation of the slit in the Roche et al. data. The larger aperture data from ISO (Moorwood et al. 1996) and Spitzer (unpublished) lie $\sim 40 \%$ above the MIDI total flux as they include the flux from the circumnuclear starburst.

\subsection{Interferometric data}

For all baselines observed, the visibility is well below the pointsource value of $V=1$, indicating that the emission region is well resolved with our interferometric resolution of $\lambda / 2 B \leq 40$ mas. As an example, we show in Fig. 3 the correlated fluxes $F_{\text {cor }}(\lambda)$ and visibilities $V(\lambda)$ obtained on 2005 Mar. 01. During this night, observations were performed using the baseline UT3 - UT4. The baseline length only increased from 50 to $60 \mathrm{~m}$, while the position angle underwent a major change from $44^{\circ}$ to $131^{\circ}$. As in the total flux, the broad dip at $10 \mu \mathrm{m}$ is due to silicate absorption.
The quality of the data at the edges of the wavelength range and in a window between 9.5 and $10.0 \mu \mathrm{m}$ (hatched region) deteriorates significantly due to atmospheric water and ozone absorption. We therefore only trust the spectra within these boundaries, that is, from $8.2 \mu \mathrm{m}$ to $9.5 \mu \mathrm{m}$ and from $10.0 \mu \mathrm{m}$ to $13.0 \mu \mathrm{m}$.

A clear increase of the correlated flux and of the visibility can be observed with increasing position angle. This increase is most pronounced in the correlated fluxes longward of $11 \mu \mathrm{m}$. We directly interpret this as an angle-dependent change of size of the emitting source: at position angles of $44^{\circ}$ to $57^{\circ}$ the correlated flux and the visibility are low, i.e. the source is more resolved and hence the emission extended, while at angles of $120^{\circ}$ and $131^{\circ}$ the correlated flux and visibility are higher, i.e. the source is less resolved and consequently the emission must be less extended. The angle-dependent size changes must be even stronger than Fig. 3 insinuates, as there is a small increase in baseline length towards larger position angles, that would lead to a decrease of the correlated flux and visibility for a round and smooth source. This is a direct and completely model independent evidence for an elongated dust structure. The elongation is perpendicular to the ionisation cone and the outflow at $\mathrm{PA}=-40^{\circ}$ in the Circinus galaxy.

To analyse the emissivity and temperature structure of the target either the visibility and the correlated flux can be used. Both approaches have advantages and disadvantages. The visibility quantifies the degree of "resolvedness" of the source, that is, it contains geometrical information on the source. The correlated flux reflects only the emission from the unresolved part of the source, which per se does not contain any geometrical information. It only receives a geometrical meaning in comparison to the total flux or to correlated fluxes at different baselines. From our observations we know that the photometry is the most uncertain part of the measurement and we have fewer independent measurements of photometries than fringe tracks. As described in Sect. 2.3, the correlated fluxes are independent of errors in the photometry; the visibility, however, always contains the photometry including its uncertainties. For the analysis and the modelling, we consider both the visibilities and the correlated fluxes as comparably useful and we will use both for our modelling of the data. Furthermore, we find that the essential inferences 


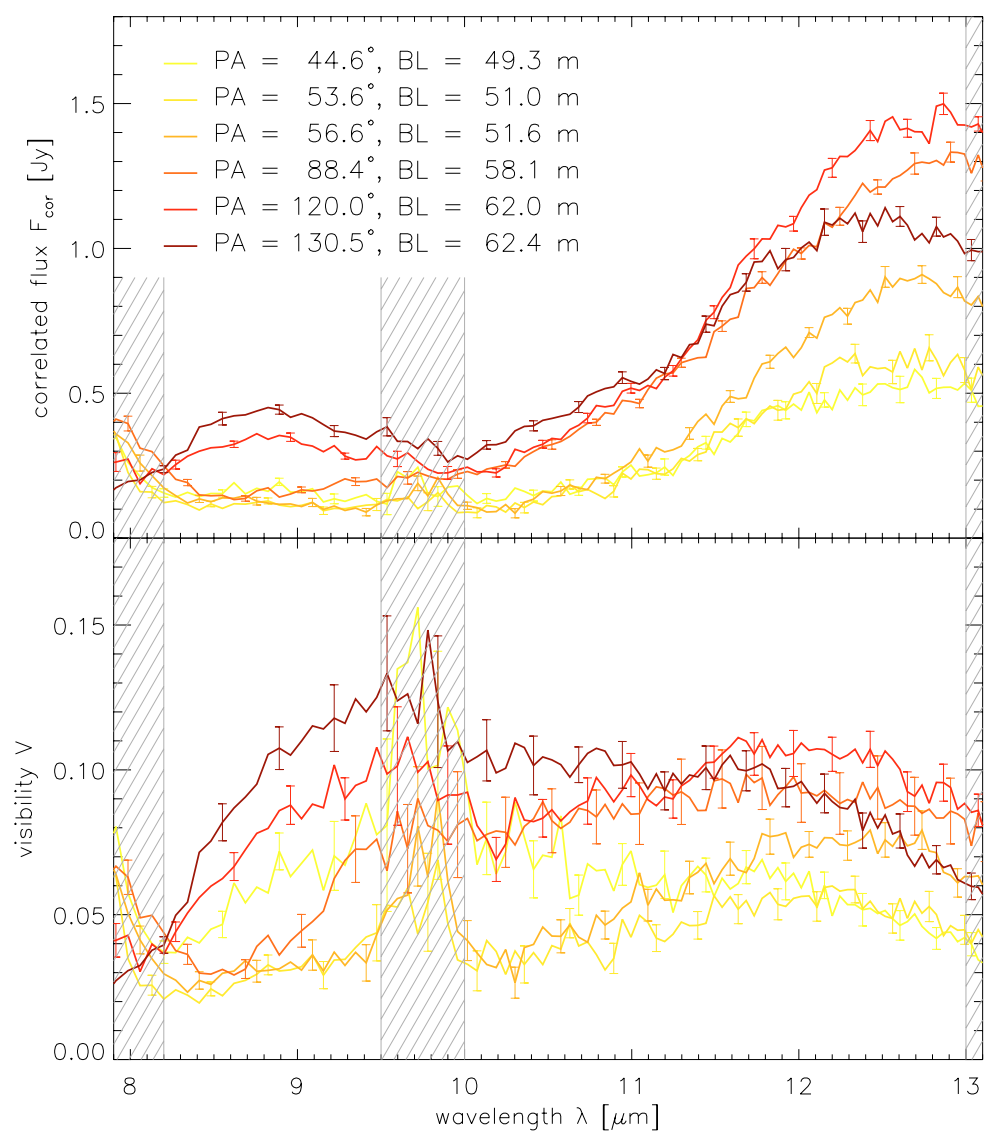

Fig. 3. Top panel: spectra of the correlated fluxes, as observed during the night of 2005 Mar. 01. Bottom panel: visibilities for the same observations. During the course of these observations, the position angle (PA) changed by $\sim 90^{\circ}$, while the projected baseline length $(B L)$ stayed roughly the same. From the angular dependence of the correlated fluxes, direct implications on the morphology can be derived: the source observed is considerably more extended in the direction of $\mathrm{PA} \sim 50^{\circ}$ than in the direction of $\mathrm{PA} \sim 130^{\circ}$. Areas affected by atmospheric absorption are hatched in grey. deduced from either the visibilities or the correlated fluxes are the same; this is reassuring.

\section{Modelling}

To interpret the visibilities in terms of a flux distribution and to deduce physical properties of the source, the data need to be fitted by models, because the $u v$ coverage is incomplete (see Sect. 2.4) and we lack absolute phase information. Our approach to model the data consists of several steps of increasing complexity, minimising any preconceptions about the source. The goal is to extract properties of the mid-infrared emission, such as size, elongation, depth of the silicate feature, etc., to sketch a picture of the nuclear dust distribution in the Circinus galaxy.

The first step of modelling is aimed at estimating the characteristic size of the emission region using a simple, one dimensional model. From the discussion in Sect. 3.2 it is clear that a one dimensional model is too simple to explain the MIR source in the nucleus of the Circinus galaxy correctly. More complex, two dimensional models are necessary. Our next step in modelling is hence a purely geometrically description of the source brightness distribution for each wavelength bin. This second, two dimensional model lead to the creation of a third, physically motivated model which also attaches physical properties to the emission region. It turns out that the latter model comprehends the main results of the first two modelling steps. For this reason, only the physical model will be presented here while the descriptions of the one-dimensional and the purely geometrical model are found in Appendices A and B.

All of the modelling was performed using the Interactive Data Language (IDL).

\subsection{Model selection}

For both the purely geometrical model as well as for the physical model we explored a multitude of geometrical shapes in order to match the data. The shapes include point sources, unifom disks, elliptical disks, elliptical Gaussian distributions and combinations thereof. We find that models including uniform disks produce results of similar $\chi^{2}$ values as Gaussian distributions. The brightness distribution of such models has sharp edges. The same is true for models including point sources (e.g. representing the hot inner edges of the torus). Sharp edges have the great disadvantage that they produce oscillations in the $u v$ plane: the $u v$ plane is not uniform, but it has many minima and maxima in a quasi periodic pattern. Additionally, phase shifts occur at the minima in the $u v$ plane. These are not observed.

For this reason, we favour uniform models with no sharp edges to describe our data. This restricts our conclusions to more general statements about the properties of the mid-infrared source of Circinus. However, we are confident that these properties are intrinsic to the source and neither an artefact of the specific model nor of the distribution of visibility points in the $u v$ plane. To be able to make clearer statements about the detailed structure of the source, a more complete coverage of the $u v$ plane is required and we are currently preparing to fill in the most obvious holes in the distribution of the visibility points, as far as this is possible with the baselines of the VLTI.

\subsection{D physical model}

The physical model consists of two concentric black body Gaussian emitters behind an extinction screen. For the absorption we used the wavelength dependency of the extinction of 
interstellar dust from Schartmann et al. (2005), but modified the profile of the silicate absorption feature in the wavelength range from 8.0 to $12.7 \mu \mathrm{m}$ using data from Kemper et al. (2004). The latter determined the feature profile towards the galactic centre and we thus expect their profile to be our best guess for the line of sight towards the nucleus of the Circinus galaxy. To obtain our template absorption profile $\tau(\lambda)$, the entire optical depth curve was normalised to 1 at the maximum absorption depth of the silicate profile: $\tau(9.7 \mu \mathrm{m})=1$.

Our two component model has the following functional form:

$$
\begin{aligned}
F(\lambda, \alpha, \delta)= & f_{1} \cdot G_{1}(\lambda, \alpha, \delta) \cdot \mathrm{e}^{-\tau_{1} \cdot \tau(\lambda)} \\
& +f_{2} \cdot G_{2}(\lambda, \alpha, \delta) \cdot \mathrm{e}^{-\tau_{2} \cdot \tau(\lambda)}
\end{aligned}
$$

where $\tau_{1}, \tau_{2}$ are the individual optical depths of the absorption screens. The scaling factors $f_{1}$ and $f_{2}$ are factors to scale the intensity of the black body Gaussian distributions $G_{1}$ and $G_{2}$. The Gaussian distributions in turn are defined by

$$
\begin{aligned}
G_{n}(\lambda, \alpha, \delta)= & \exp \left[-4 \ln 2 \cdot\left(\frac{\alpha \cos \phi+\delta \sin \phi}{r_{n} \cdot \Delta_{n}}\right)^{2}\right. \\
& \left.-4 \ln 2 \cdot\left(\frac{\alpha \sin \phi-\delta \cos \phi}{\Delta_{n}}\right)^{2}\right] \cdot F_{\mathrm{bb}}\left(T_{n}, \lambda\right)
\end{aligned}
$$

for each of the two components $n=1,2$. The parameters of the Gaussians ( $r_{1}, r_{2}$ for the axis ratios, $\Delta_{1}$ and $\Delta_{2}$ for the FWHM and $\phi$ for the orientation) are the same as for the purely geometrical model (see Appendix B), except that they are not wavelength dependent. $F_{\mathrm{bb}}$ is the black body intensity which depends on the temperature $T_{n}$. This model has 11 free parameters: the FWHM $\Delta_{n}$, the axis ratio $r_{n}$, the temperature $T_{n}$, the flux normalisation $f_{n}$ and the optical depth $\tau_{n}$ for each of the two components $n=1,2$. The common position angle $\phi$ is the eleventh parameter ${ }^{2}$. Using these parameters, the flux distributions at several wavelengths were generated and Fourier transformed. From the resulting distribution in the $u v$ plane, the visibilities corresponding to the observed baselines, $V_{i}^{\bmod }(\lambda)=\left|\mathcal{V}_{\lambda}\left(u_{i}, v_{i}\right)\right|$, were extracted. Additionally, the total flux was determined by integrating over the entire emission region: $F_{\text {tot }}^{\bmod }(\lambda)=\iint F(\lambda, \alpha, \delta) \mathrm{d} \alpha \mathrm{d} \delta$. The comparison to the observations was performed using both the correlated fluxes and the total flux, because here the precise values should match and not only the flux ratios. The correlated fluxes for the model were obtained by multiplying the modelled visibilities with the total flux of the model, i.e. $F_{i}^{\bmod }(\lambda)=V_{i}^{\bmod }(\lambda) \cdot F_{\text {tot }}^{\bmod }(\lambda)$. These could then be compared to the data. The model parameters were optimised using the Levenberg-Marquardt least-squares minimisation. The $\chi^{2}$ to be minimised was:

$\chi^{2}=\sum_{\lambda} \sum_{i=0}^{21}\left(\frac{F_{i}^{\mathrm{obs}}(\lambda)-F_{i}^{\bmod }(\lambda)}{\sigma_{F_{i}^{\mathrm{obs}}}(\lambda)}\right)^{2}$,

where the sum over $i$ is the summation over the 22 observed baseline orientations ( 21 baselines plus the total flux as a baseline with a baseline length of $B L=0 \mathrm{~m})$. The $\sigma_{F_{i}^{\text {obs }}}(\lambda)$ are the errors of the correlated fluxes.

Two fits were performed: first the model was only optimised to fit the total flux (in the following "fit 1"). In this case, several

\footnotetext{
2 Decoupling $\phi$ for the two components leads the fits to converge on a few dominant groups of visibility points only. This is the reason for keeping a single orientation angle for both components.
}

Table 2. Best fit parameters for the two component black body Gaussian models. The first column is for a fit of the total flux only (fit 1), the second column for a fit to both the total flux and the correlated fluxes (fit 2). The values in brackets were not fitted, but held fixed.

\begin{tabular}{ccc}
\hline \hline Parameter & Fit 1 & Fit 2 \\
\hline$\Delta_{1}[\mathrm{mas}]$ & 23.6 & 21.1 \\
$r_{1}$ & $(0.25)$ & 0.21 \\
$\tau_{1}$ & 1.17 & 1.18 \\
$T_{1}[\mathrm{~K}]$ & 479.6 & 333.7 \\
$f_{1}$ & $(1.00)$ & 1.00 \\
$\Delta_{2}[\mathrm{mas}]$ & 279.0 & 96.7 \\
$r_{2}$ & $(1.00)$ & 0.97 \\
$\tau_{2}$ & 2.85 & 2.22 \\
$T_{2}[\mathrm{~K}]$ & 144.4 & 298.4 \\
$f_{2}$ & $(1.00)$ & 0.20 \\
$\phi[\check{\mathrm{r}}]$ & $(60.0)$ & 60.9 \\
$\chi^{2} / N_{\text {free }}$ & 0.56 & 36.86 \\
\hline
\end{tabular}

parameters $\left(r_{1}, r_{2}, f_{1}, f_{2}\right.$ and $\left.\phi\right)$ were held fixed as they were degenerate. Then the model was fitted to the total data set including both the total flux and the 21 correlated fluxes ("fit 2 "). In case of the fit to the total flux only, there is hence no summation over $i$ in Eq. (3), as we simply had to use $i=0$. The result of the fitting process is summarised in Table 2 .

A total of 21 wavelength bins were used for the fitting, so that fit 1 has $N_{\text {free, } 1}=21 \times 1-11=10$ degrees of freedom, while fit 2 has $N_{\text {free, } 2}=21 \times 22-11=451$. The resulting total fluxes for the two models are plotted in Fig. 2 in orange (fit 1) and red (fit 2) on top of the measured total flux. The observed correlated fluxes are compared to fit 2 in Fig. 4 at two wavelengths: 8.5 and $12.5 \mu \mathrm{m}$. A comparison of fit 2 with all of the data is given in Appendix C.

The two components of this model have similar properties to those of the purely geometrical model (Appendix B): there is a smaller elongated component and a larger, nearly round component. A sketch of the result of fit 2 is depicted in Fig. 5 and the flux distribution at $11 \mu \mathrm{m}$ can be seen in Fig. 6. The smaller component has a FWHM of $\Delta_{1}=21$ mas, which corresponds to $0.4 \mathrm{pc}$ at the distance of Circinus. It is highly flattened $\left(r_{1}=0.21\right)$ and has a temperature of $T_{1}=334 \mathrm{~K}$. The distribution is found to be an optically thick black body $\left(f_{1}=1\right)$. The second component is significantly larger, as a large part of the observed flux $(\sim 90 \%)$ is resolved with our interferometric set-up. It has a FWHM of $\Delta_{2}=97$ mas, which corresponds to $2.0 \mathrm{pc}$, only a very small ellipticity and a temperature of less than $300 \mathrm{~K}$. The emissivity of the black body radiation from this component is scaled by a factor of $f_{2}=0.20$. This low scaling factor is driven by the rather extended flux distribution, which is necessary to explain the low visibilities, and the requirement not to overpredict the measured total flux. Note that the scaling factor is not to be interpreted as a geometrical covering factor. Together with the charactersitic temperature $T_{2}$, it rather is a formal description of a significantly more complex multi-temperature system. This is underlined by the fact that there is a degeneracy between the temperature and the scaling factor in the fit. When fitting only the total flux, we see a larger temperature difference between the two components than for the full fit. In both cases, fit 1 and 2, the temperatures are the least constrained parameters. We therefore consider the $330 \mathrm{~K}$ for the inner component as a lower limit for the highest dust temperature and the $300 \mathrm{~K}$ as an upper limit for the cool component. Our data rules out any significant contribution from a truly hot component with temperatures $T>1000 \mathrm{~K}$, i.e. close to the sublimation temperature of the dust. This is consistent with Prieto et al. (2004), who also 

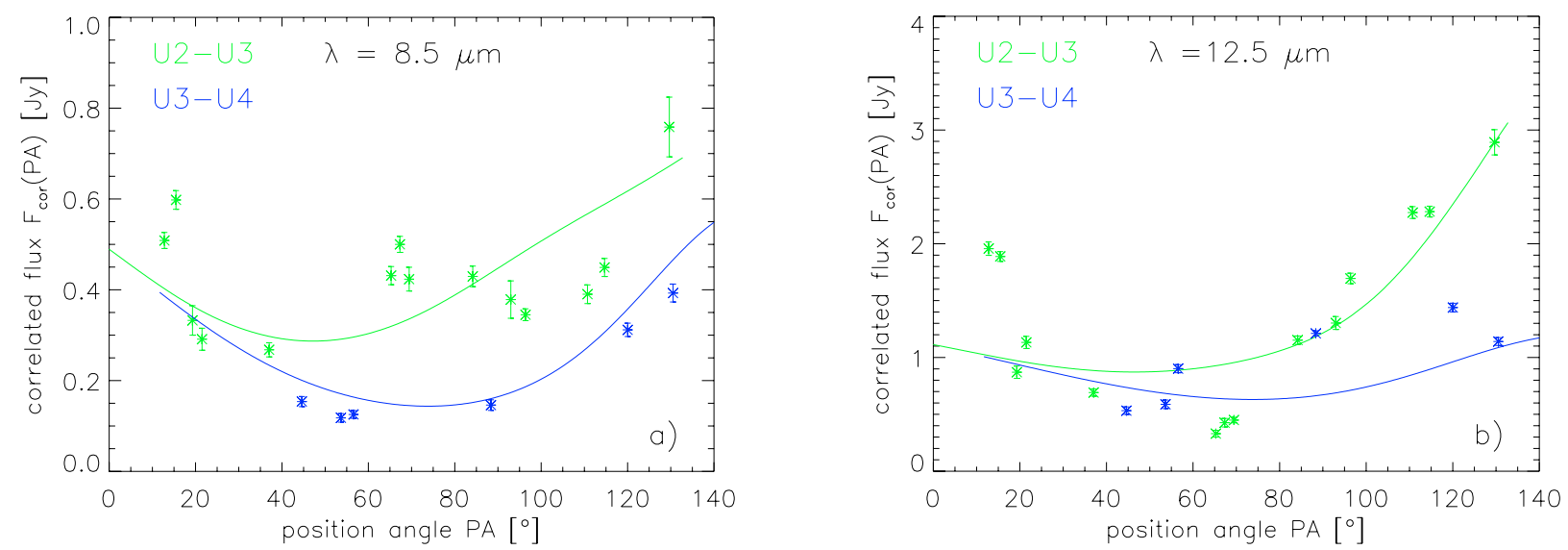

Fig. 4. Comparison of the correlated flux predicted by the physical model (fit 2 ) with the observations for two distinct wavelengths: a) 8.5 and b) $12.5 \mu \mathrm{m}$. The measured fluxes are plotted with asterisks; the continuous lines are the modelled fluxes. Note the different flux ranges for the different wavelengths.

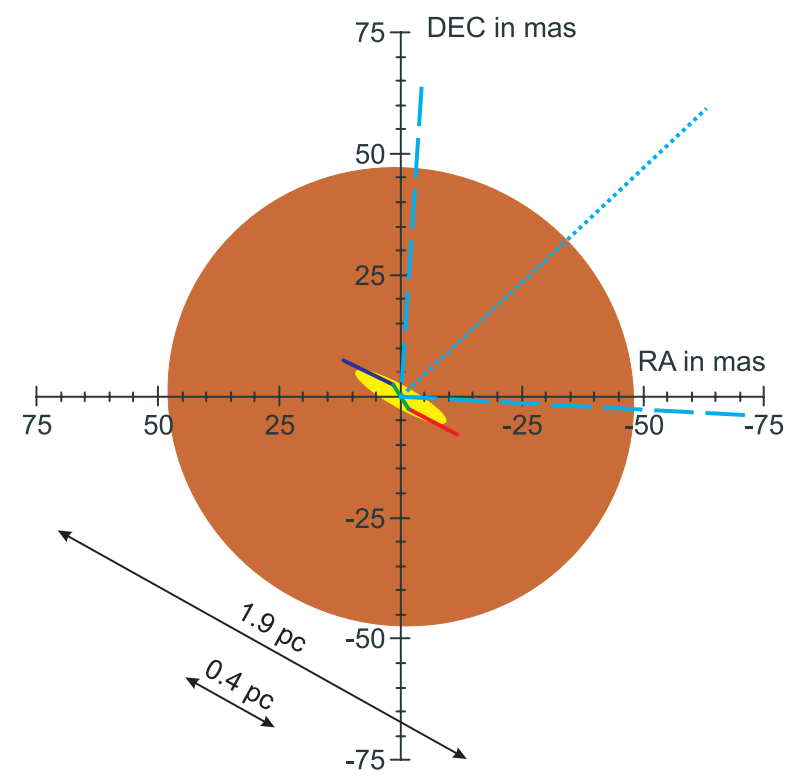

Fig. 5. Sketch of the physical model (fit 2): a highly elongated warm emission region with a temperature of $\sim 330 \mathrm{~K}$ (yellow) is surrounded by an extended, almost round and slightly cooler emission region with a temperature of $\sim 300 \mathrm{~K}$ (brown). In the centre of the sketch, the location of the $\mathrm{H}_{2} \mathrm{O}$ maser emitters in a disk from Greenhill et al. (2003) is overplotted: the blue line to the north-east traces the receeding masers and the red line to the south-west the approaching masers. The dashed light blue line traces the edge of the observed ionisation cone, the dotted line is the cone axis.

found no evidence for hot dust. The conclusion drawn from the growth of the size of the emitter with wavelength (Appendix A), namely that the temperature of the emitter decreases with increasing distance to the nucleus, is confirmed by the lower temperature of our larger component. The small component only contributes a minor fraction to the total flux. This can be seen in the top left graph of Fig. C.1: the flux contribution by the extended component is delineated by a dotted line and that by the compact disk component by a dashed line. In contrast to the observations of NGC 1068 (Jaffe et al. 2004; Poncelet et al. 2006), the correlated fluxes in the Circinus galaxy do not show a relative increase of the flux to shorter wavelengths with respect to

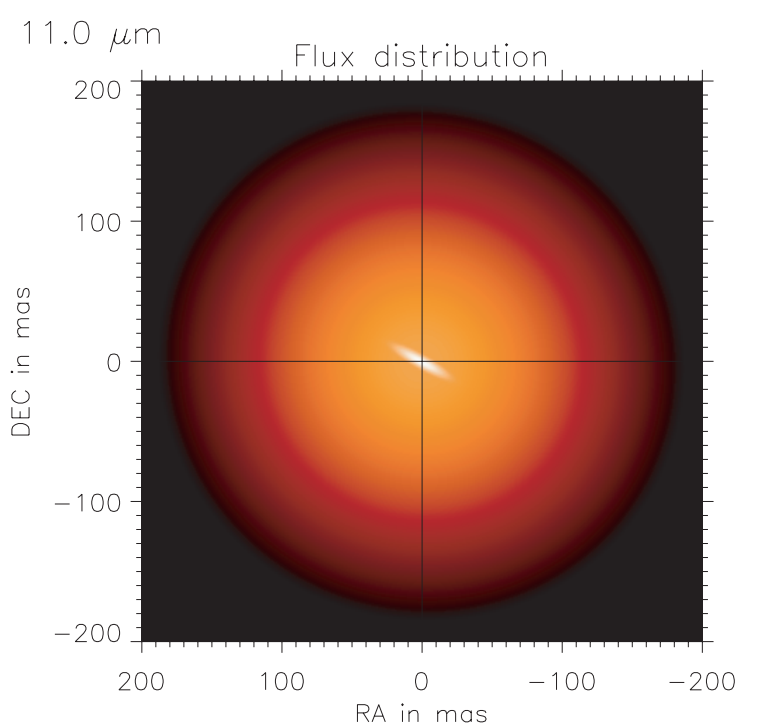

Fig. 6. Flux distribution of the physical model (fit 2) at $11 \mu \mathrm{m}$ in logarithmic scaling.

the flux at longer wavelengths. This shows that there is only a moderate increase of dust temperature towards the centre.

The model used here is a parametrised global model and does not match all aspects of the data. The reduced $\chi^{2}$ of 37 indicates that deviations well above the $5 \sigma$-level are commonly found. The aim of our model was to extract the overall properties of the emitting source. The general behaviour of the correlated fluxes for the different baseline orientations can be well reproduced, but not the details. Looking at Fig. 4, the model fluxes only show a single dip in the range of position angles traced, due to the symmetry of the object when rotated by $180^{\circ}$. The measurements exhibit considerably more rapid angular variations, rejecting a smooth axisymmetric model. The dispersed fluxes (Fig. C.1) disagree most strongly at the edges of the spectrum, at $8 \mu \mathrm{m}$, where a downturn in several of our correlated fluxes is seen, or at $13 \mu \mathrm{m}$, where the model deviates in both directions with respect to the data. As there are also small changes in the differential phase in these regions, we expect a further substructure of the source to be responsible for this unusual behaviour. 


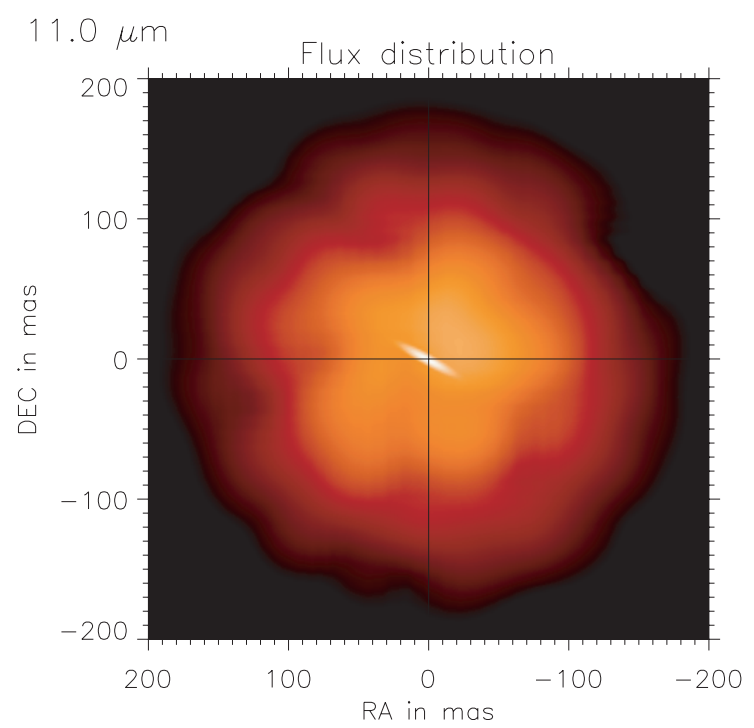

Fig. 7. Flux distribution of the physical model with added clumpiness for the extended component at $11 \mu \mathrm{m}$ in logarithmic scaling.

To verify this possibility, we undertook an additional modelling experiment: we tried to reproduce the low scaling factor of the larger component through clumpiness: instead of multiplying $F_{1}$ with the factor $f_{2}$, an uneven surface $C(\alpha, \delta)$ was used. $C(\alpha, \delta)$ can be interpreted as a screen simulating clumpiness. To make the calculation consistent, the average value of $C$ has to reproduce the scaling factor: $\langle C(\alpha, \delta)\rangle=f_{2}$. The resulting flux distribution for such a clumpy model is shown in Fig. 7. In such a simple experiment, it is possible to find clump distributions which trace the correlated flux much better (see for example Fig. 8). The reduced $\chi^{2}$ drops to values of $\chi^{2} / N_{\text {free }}<25$. This supports the assumption that such deviations are generated by a substructure of the brightness distribution.

\section{Discussion}

The modelling of our interferometric observations reveals the presence of two components of dust emission at the very centre of the Circinus galaxy: an extended $\left(r_{1}=1.0 \mathrm{pc}\right)$, warm $\left(T_{2} \sim 300 \mathrm{~K}\right)$ and fairly round emission region showing strong silicate absorption and a much smaller $\left(r_{2}=0.2 \mathrm{pc}\right)$, but only slightly warmer $\left(T_{1} \sim 330 \mathrm{~K}\right)$ component that is highly flattened and shows a less pronounced silicate absorption feature. We interpret these two components as signs for a geometrically thick "torus" of warm dust surrounding a warmer, disk-like structure (see Fig. 9). The smaller and denser disk is seen at a high inclination and it partly exhibits the silicate band in emission. It is surrounded by the larger, less dense and most likely clumpy torus component which gives rise to strong silicate absorption. The silicate absorption profiles towards both the disk and the torus component are consistent with the interstellar absorption profile observed towards the nucleus of our own galaxy. This suggests that the dust composition is the same. The result is interesting compared to that found for NGC 1068, where the dust absorption towards the nucleus clearly does not agree with the standard intergalactic absorption profile (Jaffe et al. 2004; Poncelet et al. 2006).

To understand the structure of the nuclear dust distribution in AGN, we developed hydrodynamical models of dusty tori (for a detailed description see Schartmann 2007). These models indeed also show a disk-like structure in the inner region which is surrounded by a geometrically thick "torus". The torus is not continuous but has a filamentary structure (rather than clumpy) and the disk is turbulent.

In the following, we will develop a picture of the central parsec region in Circinus which aims to explain the results of the MIDI measurements in the context of the hydrodynamical model. In addition, the picture needs to be consistent with the previously known properties of this galactic nucleus.

\subsection{Extinction}

To interpret the nuclear emission from Circinus and the silicate absorption in the torus, we need to estimate the foreground extinction. Radiation from the nuclear region of Circinus suffers extinction from two main foreground absorbers: our own galaxy (Circinus is located at a galactic latitude of $b=-4^{\circ}$ ) and the intrinsic absorption in the galactic disk of the Circinus galaxy.

By observing the reddening of several stars in the vicinity of Circinus, Freeman et al. (1977) estimate the visual extinction due to our own galaxy to be only $A_{V}=1.50 \pm 0.15 \mathrm{mag}$, as Circinus lies in a window of low galactic extinction. The extinction towards the nuclear region within Circinus itself is much less constrained and estimates from near-infrared colors vary from $A_{V}=6 \mathrm{mag}$ for a foreground screen to $A_{V} \gtrsim 20 \mathrm{mag}$ for a mix of dust and stars (Prieto et al. 2004; Mueller Sánchez et al. 2006). In the latter case, the colours saturate so that higher extinctions cannot be ruled out. The near-infrared observations which underlie this estimate probe a region on scales of less than $10 \mathrm{pc}$ distance from the nucleus. Given the presence of a nuclear starburst (Mueller Sánchez et al. 2006), a mix of dust and stars appears to be more realistic and we adopt a foreground extinction, between us and $\sim 2 \mathrm{pc}$ from the nucleus, of $A_{V} \sim 20 \mathrm{mag}$.

From the optical depth of the silicate feature seen in the extended torus component, we can estimate the extinction towards those regions of the torus where the warm dust emission arises. Our value of $\tau_{2} \sim 2.2$ for the optical depth is at the lower end of the values found by Roche et al. (2006), who cite a range of $2.2 \leq \tau_{\text {sil }} \leq 3.5$, depending on where they extract the spectrum along their slit. Given the low spectral resolution of MIDI the minimum of the absorption trough may be washed out, leading to lower optical depths. We therefore consider the two values to be in good agreement, although our observations rule out $\tau_{\text {sil }}>3.0$ for the nucleus. In the following, we will assume $\tau_{\text {sil }}=2.5$ towards the larger $(r=1 \mathrm{pc})$ torus component.

We use the opacity scaling of Schartmann et al. (2005), to convert the opacity measured in the $9.5 \mu \mathrm{m}$ silicate feature to an equivalent visual extinction, $\tau_{V}=12.2 \cdot \tau_{\text {sil }}$. Hence, the absorption in the visual is $A_{V}=1.09 \cdot \tau_{V} \mathrm{mag}=13.3 \cdot \tau_{\text {sil }}$ mag. For $\tau_{\text {sil }}=2.5$, we obtain $A_{V}=33 \mathrm{mag}$, which means that there are an additional 11 mag of extinction towards the torus region compared to the limit derived from the near-infrared colours. Most likely, the extinction towards the accretion disk itself is even higher.

In contrast to the MIDI observations of NGC 1068 (Jaffe et al. 2004; Poncelet et al. 2006), we do not observe a further increase of the silicate absorption feature when zooming into the nucleus with our interferometric observations. Instead, the absorption feature appears to be less pronounced in the correlated fluxes, which can also be inferred directly from the convex shape of the visibilities (e.g. from Fig. 3). In general, the silicate feature is expected to appear in emission for lines of sight, where the (appropriately high) temperature decreases away from 

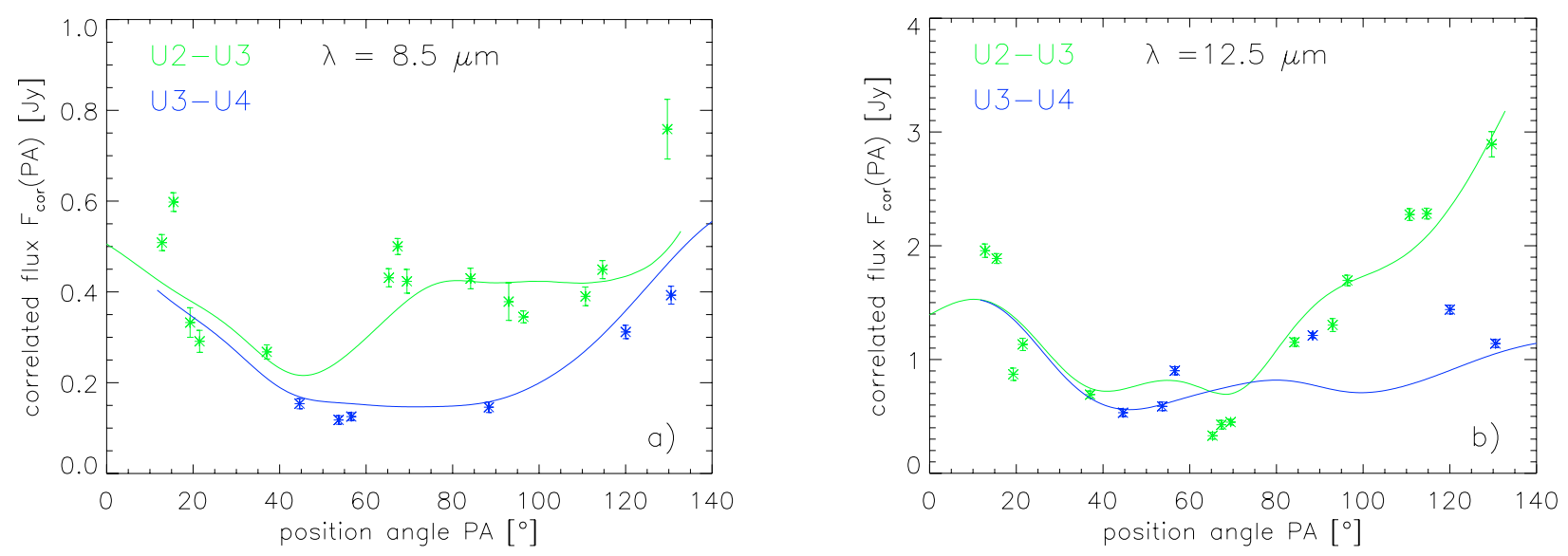

Fig. 8. Correlated flux of the physical model with simulated clumpiness for the extended component: a) 8.5 and b) $12.5 \mu \mathrm{m}$. The measured fluxes are plotted with asterisks; the continuous lines are the modelled fluxes. The clumpiness introduces significant changes in the correlated fluxes compared to the smooth model (see Fig. 4). From among 1000 clumpy models we found at least one (shown here), which matches the data well. This shows that clumpiness of a certain geometry can greatly improve the fit to the data.

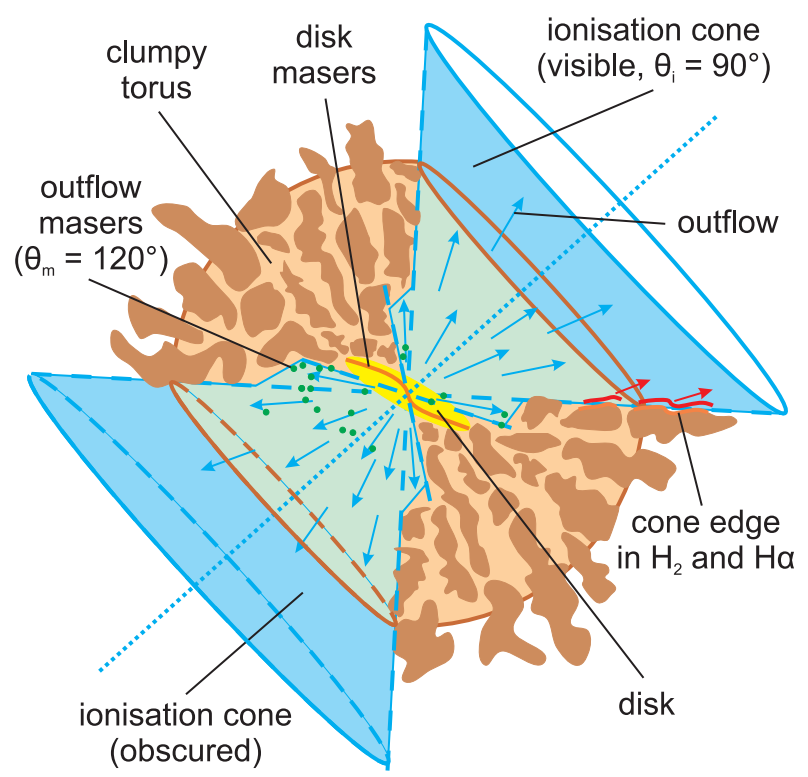

Fig. 9. Cut through the dusty torus in the central parsec-sized region of the Circinus galaxy derived from the interferometric observations in the MIR. See text for details.

the observer. This is the case for the directly visible inner rim of the torus and, therefore, expected and observed for Seyfert $1 \mathrm{ob}-$ jects. In the opposite case (cold dust along the line of sight), the silicate feature will appear in absorption, as seen in Seyfert 2 objects. A decrease of the silicate feature in absorption therefore means that more and more of the Seyfert 1 type silicate emission is able to shine through the torus body. These inferences are consistent with a hidden Seyfert 1 nucleus in Circinus, a fact also suggested by the observation of broad emission lines in polarised light (Oliva et al. 1998).

In summary, we conclude that the obscuration on our line of sight towards the inner parsec of Circinus is composed of the following three extinction components: extinction by our Milky Way, $A_{V}(\mathrm{MW})=1.5 \mathrm{mag}$, by the foreground dust within Circinus, $A_{V}$ (disk) $=20 \mathrm{mag}$, and by the nuclear dust, $A_{V}$ (nuclear) $=11 \mathrm{mag}$.

\subsection{Energy budget}

The energy emitted by the source in the $N$ band can be estimated by integrating the flux in the dereddened spectrum, giving a luminosity in the $N$ band of $L_{7.5-13.5 \mu \mathrm{m}}=1.3 \times 10^{9} L_{\odot}=$ $5.1 \times 10^{35} \mathrm{~W}$

To estimate the total luminosity of the dust emission, we integrate under the flux of our physical model (fit 2) from $v=0$ to $\infty$ without applying any absorption, that is $\tau_{1}=\tau_{2}=0$. Orientation effects only affect significantly the smaller disk component. A correction by assuming oblate spheroids seen edge-on leads to an increase of the flux by about $20 \%$. With this method, we obtain a total luminosity from the dust of $L_{\text {dust }}=4.1 \times 10^{9} L_{\odot}$. This is four times more than the luminosity measured in the $N$ band alone. Considering that we have not traced the contribution by dust at $T \lesssim 150 \mathrm{~K}$, we round our value up to $L_{\text {dust }} \sim 5 \times 10^{9} L_{\odot}$ as our best estimate for the luminosity of the nuclear dust at $r<2$ pc in the infrared.

We can take this value to estimate the luminosity of the accretion disk, $L_{\text {acc }}$, which presumably is responsible for heating the dust. In doing so, we have to take into account that not all of the accretion luminosity is available for heating the torus. A significant fraction of $L_{\text {acc }}$ will escape along the directions of the ionisation cones. With an opening angle of $90^{\circ}$ (Veilleux \& Bland-Hawthorn 1997; Wilson et al. 2000), the total solid angle covered by the torus, as seen from the centre, is $2 \sqrt{2} \pi$. This corresponds to about 0.7 of the full sphere. Assuming a radiation characteristic for an optically thick accretion disk which is proportional to $\cos \theta$, about half of the radiation is emitted in direction of the dust in the torus. That is, only half of the energy from the accretion disk will be absorbed by the dust and re-radiated in the infrared. If we thus assume $L_{\text {dust }} \sim 0.5 \cdot L_{\mathrm{acc}}$, this leads to a luminosity of the central energy source of $L_{\mathrm{acc}} \sim 10^{10} L_{\odot}$.

Consistent with our estimate, Moorwood et al. (1996) also report the luminosity of the nucleus to be $\sim 10^{10} L_{\odot}$. Their integration under the UV bump (the unobscured radiation) yields the same luminosity as we observed in the MIR: $L_{\mathrm{UV}}=5 \times$ $10^{9} L_{\odot}$. Oliva et al. (1999) cite a total ionising radiation of the 


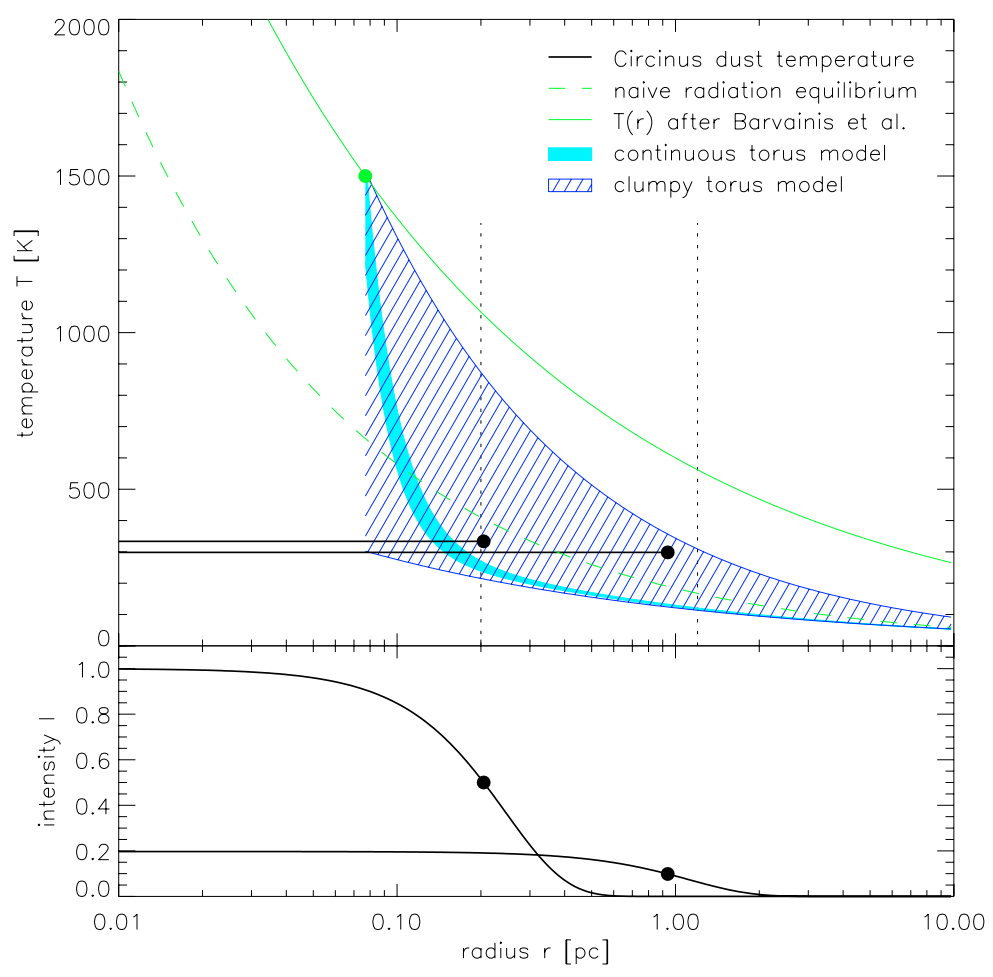

Fig. 10. Radial temperature gradients in the central dust distribution. The radial temperature distribution of our simple "physical" model (thick black continuous lines) is compared to the temperature distributions for a clumpy (hatched area) and continuous torus (light blue / grey filled area) as well as to the temperature dependency of dust directly illuminated by the central energy source (green / grey lines). The point where the sublimation temperature of the dust, $T_{B}(r)=1500 \mathrm{~K}$, is reached is marked by a green / grey circle. The lower part of the graph shows the intensity of the Gaussian components (depending on the scaling factor $f$ ). The HWHM of the Gaussian components are marked by circles. The scales probed by our interferometric set-up lie within the dotted vertical lines. nucleus on the order of $L_{\text {ion }}=1-4 \times 10^{10} L_{\odot} \cdot \sin ^{-2} i$, where $i$ is the inclination angle of the ionisation cone with respect to our line of sight. For $i>60$ (Elmouttie et al. 1998), this translates into $1-7 \times 10^{10} L_{\odot}$. Luminosities of the AGN in Circinus significantly higher than $10^{10} L_{\odot}$ can be ruled out, as this would imply a higher MIR flux than observed. The currently derived intrinsic 2 to $10 \mathrm{keV}$ luminosities of the nuclear X-ray source are all below $5 \times 10^{8} L_{\odot}$, which is well below the energy needed to heat the dust. The dust is mainly heated by soft X-rays and UV radiation rather than by radiation in the 2 to $10 \mathrm{keV}$ band alone.

Finally, we can compare our luminosity estimate for the accretion disk to the Eddington luminosity. For a black hole with a mass of $M_{\mathrm{BH}}=1.7 \times 10^{6} M_{\odot}($ Greenhill et al. 2003), this luminosity is $L_{\mathrm{E}}=5.6 \times 10^{10} L_{\odot}$. With our value of $L_{\mathrm{acc}} \sim 10^{10} L_{\odot}$, we find Circinus to accrete at $18 \%$ of its Eddington luminosity.

\subsection{Temperature dependency and clumpiness}

Less than a parsec from this central energy source, the dust is most likely heated by the radiation from the central accretion disk alone and not by a homogeneous energy input, such as by a starburst. The discussion of the energy budget of the nucleus has shown that no further energy source is necessary. A heating by a nuclear energy source is also suggested by the decrease of the dust temperature to the outside.

The temperature decrease we observe is, however, very shallow and the temperature of our large component is relatively high considering the distance of its dust to the heating source: the dust has a temperature of $\sim 300 \mathrm{~K}$ out to distances of $1 \mathrm{pc}$ from the nucleus. To investigate the implications of this finding on the properties of the dust distribution, we compare the dust temperature in Circinus to the temperature expected for a direct illumination of the dust and to the temperature inferred from our radiative transfer modelling of continuous and clumpy AGN tori.
The various temperature dependencies are shown in Fig. 10 and we will discuss them one by one in the following.

Assuming a naive radiation equilibrium, the temperature of a dust particle at the distance $r$ from the illuminating source with the luminosity $L_{\text {acc }}$ is given by

$T_{\mathrm{N}}(r)=194\left(\frac{L_{\mathrm{acc}}}{r^{2}} \frac{\mathrm{pc}^{2}}{10^{10} L_{\odot}}\right)^{1 / 4} \mathrm{~K}$

Taking into account the absorption and emission characteristics of the dust grains leads to a more realistic description of the temperature dependency (Barvainis 1987). For emission in the infrared, the temperature dependency then reads:

$T_{\mathrm{B}}(r)=624\left(\frac{L_{\mathrm{acc}}}{r^{2}} \frac{\mathrm{pc}^{2}}{10^{10} L_{\odot}}\right)^{1 / 5.6} \mathrm{~K}$.

The two temperature dependencies $T_{\mathrm{N}}(r)$ and $T_{\mathrm{B}}(r)$, calculated for $L_{\text {acc }}=10^{10} L_{\odot}$, are plotted Fig. 10 by a green dashed line and a green continuous line respectively (in the printed version the green lines appear grey). The realistic case leads to significantly higher temperatures than the naive case, as the individual dust particles reradiate the energy less effectively than a black body. Here, we have not taken into account the anisotropic radiation characteristic of the central energy source, the accretion disk, which emits less energy in direction of the dust distribution than in direction of the ionisation cones (see Sect. 5.2). This leads to a lower temperature of the dust near the plane of the disk, so that $T_{B}(r)$ should be considered as an upper limit for the dust temperature at a certain radius.

In the case of a continuous dust distribution, the temperature is expected to drop steeply outside the inner funnel of the torus, where the dust is directly illuminated by the central source. The dust at larger radii is shielded from the nuclear heating and by consequence at lower temperatures. For a clumpy torus, the 
temperature dependency should be somewhat intermediate between the continuous case and the radiation equilibrium for totally unobscured lines of sight, that is, $T_{\mathrm{B}}(r)$. The behaviour is reproduced by our radiative transfer modelling of AGN tori: the shaded areas show the temperature ranges (from mean to maximum temperature) of the individual cells in a continuous and a clumpy torus model (for a detailed description of the modelling we refer to Schartmann et al. 2007). For this purpose, the radii were rescaled so that the inner radii of the torus, where the sublimation temperature is reached, coincide with the sublimation radius predicted by $T_{B}(r)$. As expected, the continuous model (filled, light blue / grey area) has a very strong drop in the temperature behind the inner edge of the dust distribution, while the clumpy model (dark blue, hatched area) has a much wider temperature range, including lower temperature clouds at small distances from the nucleus and higher temperatures at larger distances.

Finally, the thick black continuous lines trace the temperatures of the two dust components in Circinus. The lines are drawn out to the half width at half maximum (HWHM) of the respective component. From the figure it is obvious that the temperatures seen by MIDI disagree with a continuous dust distribution as the temperatures lie significantly above the distribution and no sharp temperature rise to the centre is seen. The high temperatures at large distances from the nucleus can only be achieved when clouds also at larger distances have free lines of sight to the heating source. As the temperatures lie within the temperature range for a clumpy dust distribution, we conclude that the dust most likely is distributed in a clumpy medium. All our temperatures lie below those of $T_{B}(r)$, as expected for partial shadowing of the clouds.

There are several further indications for a "clumpy" or patchy dust distribution in our data:

The low emissivity filling factor of the torus-like component in our physical model is required by the data and best explained by an inhomogeneity of the emitting dust. Patchy emission appears the only way for thermal emission of dust at $T \sim 300 \mathrm{~K}$ to be as extended as observed here, while at the same time being consistent with the total flux of the source.

The most compelling evidence, however, comes from the behaviour of the correlated fluxes (a) with wavelength or (b) with changing baseline (for the sake of simplicity we will only refer to the correlated fluxes $F_{\text {cor }}$ in this paragraph, nevertheless the same statements are true for the behaviour of the visibilities $V$ ). The behaviour observed cannot be explained by a smooth brightness distribution.

When considering the set of all visibility points observed at a single wavelength, i.e. $F_{\mathrm{cor}, \lambda}(\mathrm{PA})$, the need for clumpiness becomes obvious when comparing Fig. 4 to Fig. 8, as was already discussed in Sect. 4.2. We have also compared the variations we observe in our data to those that can be seen in the correlated fluxes of our clumpy radiative transfer models (Schartmann et al. 2007): when we calculate the correlated fluxes of such a torus model for different position angles, $F_{\mathrm{cor}, \lambda}(\mathrm{PA})$, we obtain variations of up to $50 \%$ of the flux, similar to those shown in Fig. 8. The variations become stronger for longer baselines, where substructures are better resolved.

Similarly, the need for a clumpy structure becomes obvious when considering the individual visibility points at different wavelengths, that is, the dependence of the correlated flux over the $N$ band, $F_{\text {cor,PA }}(\lambda)$. In this case, we observe wiggles in the correlated fluxes that can only be reproduced by a substructure, e.g. the downturn at $\lambda>12 \mu \mathrm{m}$ for $\mathrm{BL} \sim 36 \mathrm{~m}, \mathrm{PA} \sim 67^{\circ}$ (see Fig. C.1).
An inhomogeneous dust distribution complicates the problem of determining the morphology of the source significantly, when interferometric methods are used. With the current $u v$ coverage, we are not able to say anything about the details of the clump distribution of the source. More data are needed to disentangle these and also to enable us to trace how the morphology changes with the wavelength.

\subsection{Orientation and geometry of the dust distribution}

Both direct inspection of the data (see Fig. 3) and our modelling reveal that the source is elongated in direction of PA $60^{\circ}$. This effect is dominated by our observations with longer baselines (UT3 - UT4); the shorter baseline observations (UT2 - UT3) do not show a clear orientational preference. In our interpretation, this means that the inner component is more disk-like, while the outer component traces an almost spherical or very thick toroidal component.

The projected orientation of our disk component is in very good agreement with the orientation of several components in this AGN which are directly related to the nuclear dust distribution: its axis coincides with the direction of the ionisation cone, which points towards $\mathrm{PA}=-44^{\circ}$ (Maiolino et al. 2000; Wilson et al. 2000) and the bidirectional outflow observed in $\mathrm{CO}$ pointing towards $\mathrm{PA}=124^{\circ}$ and $\mathrm{PA}=-56^{\circ}$ (Curran et al. 1998). Our measurements are hence the first direct confirmation for the presence of a dust structure extended perpendicularly to the outflow and the ionisation cone in an AGN without taking any previous knowledge on the source into account.

The nucleus of Circinus is special in another feature: it displays a very strong emission of water vapour masers. This emission was first detected by Gardner \& Whiteoak (1982). A detailed study was performed by Greenhill et al. (2003): the VLBI observations of the $6_{16}-5_{23}$ transition of $\mathrm{H}_{2} \mathrm{O}$ show an $\mathrm{S}$ shaped locus and a wider distribution of maser sources at the nucleus of the Circinus galaxy. The authors attribute the sources detected to two distinct populations: a warped, edge-on "accretion disk" and a wide angle outflow. The warped disk has an inner radius of $(0.11 \pm 0.02) \mathrm{pc}$ and extends out to $\sim 0.4 \mathrm{pc}$. These are the only observations at the same or even better angular resolution as ours. An almost perfect alignment is found between the outer radius of the maser disk with a position angle of $\mathrm{PA}=56 \pm$ $6^{\circ}$ (Greenhill et al. 2003) and our disk component (cf. Figs. 5 and 9$)$.

Because of the high axis ratio of $r_{1}=0.21$, our observations suggest that the disk component is nearly edge-on: $i_{\text {disk }}>60^{\circ}$. If it were a truly edge-on disk $\left(i=90^{\circ}\right)$, it would then have to be warped or to have a thickness of $h / r=r_{1}$ to appear as we observe it. When observed edge-on, the maser disk with a warp of $27^{\circ}$ can easily produce the same extent as observed for our smaller component. Considering this and the good agreement of the position angles, we believe the maser emission originates in the dense dusty and molecular medium associated with our disk component ${ }^{3}$.

From the present data, we cannot make any detailed statements concerning the shape of the larger torus component, as its proportions are not very well constrained. It must be significantly extended also in the direction of the ionisation cone and

\footnotetext{
3 Note that we are not able to determine an accurate position of the MIR source, because high resolution astrometry is not possible with MIDI. We hence assume that the torus emission is centered on the maser disk, although it might well be possible that there is an offset for a slightly inclined torus.
} 
the measurements are consistent with an almost circular dust distribution when seen in projection. When seen almost edge-on, this implies $h / r \sim 1$. The component can, however, not be entirely spherical but it must have an opening angle of $90^{\circ}$ for the ionisation cones (see Fig. 9). From our side-on viewing position, we see a projection of this hollowed spherical dust structure, which leads to its almost circular appearance. More measurements at shorter baselines are necessary to provide better constraints on the distribution of the more extended dust emission.

\subsection{Collimation of the ionisation cone and outflow}

In the context of the two components, namely the disk and the torus, it is interesting to address the question which component causes the collimation of the outflow and the ionisation cone. The latter has an opening angle of $\theta_{\mathrm{i}}=90^{\circ}$ and a sharp boundary seen clearest in [O III] and $\mathrm{H} \alpha$ (Wilson et al. 2000). The same opening angle is found for the extended X-ray emission, overlapping with the ionisation cone (Smith \& Wilson 2001) as well as for the CO outflow (Curran et al. 1999). Finally, the high ionisation lines in direction of the ionisation cone reported by Maiolino et al. (2000) ([Si VI]) and Prieto et al. (2004) ([Si VII]) show an even smaller opening angle. Summarised, this means that at scales $>2$ pc only $30 \%$ of the solid angle are exposed directly to radiation from the nuclear engine. This cannot be explained by the $\cos \theta$ radiation characteristic of the accretion disk, especially considering the sharp boundaries of the cone. On scales less than $1 \mathrm{pc}$, Greenhill et al. (2003) found a wide angle outflow of maser emitters. It is supposed to cover 80 to $90 \%$ of the solid angle, corresponding to an opening angle of $\theta_{\mathrm{m}}=120^{\circ}$. This is consistent with a collimation of the outflow by a disk with a warp of $27^{\circ}$. These observations lead to the conclusion that the large torus component extending from $\sim 0.2$ to $1.0 \mathrm{pc}$ must be responsible for the further collimation of the wide angle outflow to the narrow one observed at larger scales (see Fig. 9).

Further support for this picture comes from the observation of molecular hydrogen gas just below the ionisation cone on scales from 6 pc (Mueller Sánchez et al. 2006) up to $20 \mathrm{pc}$ (Maiolino et al. 1998, 2000). This gas most likely is part of the cooler regions of the dusty torus. Once clouds enter the ionisation cone and are directly irradiated by the central source, they emit $\mathrm{H} \alpha$ and [Si VI] emission, which is observed in the ionisation cone and which is especially strong on its southern edge. The clouds are then accelerated and entrained by the outflow (for a more detailed discussion, see Packham et al. 2005).

As an alternative to the thick toroidal dust distribution in unified models, warped disks are often put forward. Fully in line with this, Greenhill et al. (2003) have claimed that the warped disk alone may be responsible for the obscuration of the nuclear source and for the collimation of the outflow in Circinus, obviating the need for a geometrically thick dust distribution. Our observations, however, have added the decisive component: they have proven that a geometrically thick dust distribution is indeed present in the nucleus of Circinus. It is obvious that the opening angle observed at larger scales is too narrow to be produced by merely a disk with a warp of $27^{\circ}$. Hence the disk component cannot be the source of the collimation for the outflow and ionising radiation alone.

From our observations we cannot say anything about cooler dust, with temperatures $T<100 \mathrm{~K}$, and it is likely that the structure we observe is embedded in a larger component of cool gas and dust extending out to the starburst on scales of $10 \mathrm{pc}$.

\section{Conclusions}

We have obtained extensive interferometric observations of the nucleus of the Circinus galaxy covering a wavelength range from 8.0 to $13.0 \mu \mathrm{m}$, using the MIDI interferometer at the VLTI. Through direct analysis of the data and several steps of modelling with increasing complexity, we have arrived at the following conclusions for the nuclear dust distribution in this active nucleus:

1. The distribution of dust cannot be described by a single, simple component. However, it appears to be distributed in two components:

(a) a dense and warm $\left(T_{1} \gtrsim 330 \mathrm{~K}\right)$ disk component with a radius of $0.2 \mathrm{pc}$ and

(b) a less dense and slightly cooler $\left(T_{2} \lesssim 300 \mathrm{~K}\right)$ geometrically thick torus-like component extending out to $1 \mathrm{pc}$ half-light radius.

2. This disk-torus configuration is oriented perpendicularly to the ionisation cone and to the outflow.

3. The compact, disk-like dust component coincides in orientation and extent with a nuclear maser disk.

4. From the total energy needed to heat the dust and from the solid angle intercepted by the dust torus, we infer a luminosity of the accretion disk of $L_{\text {acc }} \approx 1 \times 10^{10} L_{\odot}$. This corresponds to $\sim 20 \%$ of the Eddington luminosity of the $M_{\mathrm{BH}}=1.7 \times 10^{6} M_{\odot}$ nuclear black hole.

5. The properties of the larger torus component are less constrained by the data. With an $h / r \sim 1$, it is consistent with a thick, edge-on torus which has cavities for the ionisation cone and outflow. This component is most likely responsible for collimating the radiation and the wide angle outflow originating in the nucleus down to the observed opening angle of the ionisation cone and outflow of $90^{\circ}$ at larger distances.

6. We find strong evidence for a clumpy or filamentary dust distribution in the torus from three lines of reasoning:

(a) The large component only has a low effective scaling factor of $\sim 20 \%$.

(b) The visibility measurements show an irregular behaviour that can be explained by clumpiness.

(c) The radial temperature dependency of the dust, $T(r)$, has a rather shallow decrease which requires that some of the outer dust is directly exposed to the nuclear radiation.

7. The silicate absorption depth is less pronounced for the disk component than for the extended component, arguing for a $10 \mu \mathrm{m}$ silicate feature in emission in the innermost, diskshaped regions of the dust distribution.

8. The silicate absorption profile is consistent with that of normal galactic dust. We hence see no evidence for dust reprocessing. This might be connected to the moderate maximum dust temperatures, $T_{\max } \lessgtr 400 \mathrm{~K}$.

Taking this evidence together, we conclude, that the long postulated dust torus exists in this Seyfert 2 nucleus. It is, however, significantly more fine structured than the doughnut-shaped models which have been proposed as long as no spatially resolved information was available.

Acknowledgements. We thank Roy van Boekel for providing his calibrator templates. We are very grateful to the anonymous referee for his helpful comments and a quick refereeing process. KT thanks the MPI für Astronomie and especially $\mathrm{KM}$ for the opportunity to work on this exciting project as part of his Ph.D. thesis. 


\section{References}

Arsenault, R., Alonso, J., Bonnet, H., et al. 2003, in Adaptive Optical System Technologies II, ed. P. L. Wizinowich, \& D. Bonaccini, Proc. SPIE, 4839, 174

Barvainis, R. 1987, ApJ, 320, 537

Bonaccini, D., Farinato, J., Comin, M., et al. 2000, in Adaptive Optical Systems Technology, ed. P. L. Wizinowich, Proc. SPIE, 4007, 431

Curran, S. J., Johansson, L. E. B., Rydbeck, G., \& Booth, R. S. 1998, A\&A, 338, 863

Curran, S. J., Rydbeck, G., Johansson, L. E. B., \& Booth, R. S. 1999, A\&A, 344, 767

Elmouttie, M., Haynes, R. F., Jones, K. L., Sadler, E. M., \& Ehle, M. 1998, MNRAS, 297, 1202

Freeman, K. C., Karlsson, B., Lynga, G., et al. 1977, A\&A, 55, 445

Gardner, F. F., \& Whiteoak, J. B. 1982, MNRAS, 201, 13

Greenhill, L. J., Booth, R. S., Ellingsen, S. P., et al. 2003, ApJ, 590, 162

Jaffe, W., Meisenheimer, K., Röttgering, H. J. A., et al. 2004, Nature, 429, 47

Jaffe, W. J. 2004, in New Frontiers in Stellar Interferometry, ed. W. A. Traub (Bellingham, WA: The International Society for Optical Engineering), Proc. SPIE, 5491, 715

Kemper, F., Vriend, W. J., \& Tielens, A. G. G. M. 2004, ApJ, 609, 826 Leinert, C., Graser, U., Przygodda, F., et al. 2003, Ap\&SS, 286, 73

Maiolino, R., Krabbe, A., Thatte, N., \& Genzel, R. 1998, ApJ, 493, 650

Maiolino, R., Alonso-Herrero, A., Anders, S., et al. 2000, ApJ, 531, 219

Matt, G., Fiore, F., Perola, G. C., et al. 1996, MNRAS, 281, L69
Moorwood, A. F. M., Lutz, D., Oliva, E., et al. 1996, A\&A, 315, L109 Mueller Sánchez, F., Davies, R. I., Eisenhauer, F., et al. 2006, A\&A, 454, 481 Oliva, E., Salvati, M., Moorwood, A. F. M., \& Marconi, A. 1994, A\&A, 288, 457

Oliva, E., Marconi, A., Cimatti, A., \& Alighieri, S. D. S. 1998, A\&A, 329, L21 Oliva, E., Marconi, A., \& Moorwood, A. F. M. 1999, A\&A, 342, 87

Packham, C., Radomski, J. T., Roche, P. F., et al. 2005, ApJ, 618, L17

Poncelet, A., Perrin, G., \& Sol, H. 2006, A\&A, 450, 483

Prieto, M. A., Meisenheimer, K., Marco, O., et al. 2004, ApJ, 614, 135

Roche, P. F., Aitken, D. K., Smith, C. H., \& Ward, M. J. 1991, MNRAS, 248, 606

Roche, P. F., Packham, C., Telesco, C. M., et al. 2006, MNRAS, 367, 1689

Ruiz, M., Efstathiou, A., Alexander, D. M., \& Hough, J. 2001, MNRAS, 325, 995

Schartmann, M. 2007, Ph.D. Thesis, Max-Planck-Institute for Astronomy, Heidelberg, Germany

Schartmann, M., Meisenheimer, K., Camenzind, M., Wolf, S., \& Henning, T. 2005, A\&A, 437, 861

Schartmann, M., Meisenheimer, K., Camenzind, M., et al. 2007, A\&A

Siebenmorgen, R., Moorwood, A., Freudling, W., \& Kaeufl, H. U. 1997, A\&A, 325,450

Smith, D. A., \& Wilson, A. S. 2001, ApJ, 557, 180

Swain, M., Vasisht, G., Akeson, R., et al. 2003, ApJ, 596, L163

Veilleux, S., \& Bland-Hawthorn, J. 1997, ApJ, 479, L105

Wilson, A. S., Shopbell, P. L., Simpson, C., et al. 2000, AJ, 120, 1325

Wittkowski, M., Kervella, P., Arsenault, R., et al. 2004, A\&A, 418, L39 
K. R. W. Tristram et al.: The nuclear dust torus in the Circinus galaxy, Online Material $p 1$

\section{Online Material}


K. R. W. Tristram et al.: The nuclear dust torus in the Circinus galaxy, Online Material $p 2$ 


\section{Appendix A: 1D model}

With this first step of modelling, we aim to estimate the characteristic size of the emission region. To this end, we assumed the source to be axisymmetric on the plane of the sky and plotted all visibility measurements, regardless of the position angle, as a function of the baseline length $B L: V_{\lambda}(B L)$. This can be done independently for all wavelength bins measured by MIDI. Examples for such a plot are shown for two wavelength bins at $8.5 \mu \mathrm{m}$ and $12.5 \mu \mathrm{m}$ in the top row of Fig. A.1. The visibility estimates show a decrease towards longer baselines from $V=1(B L=0 \mathrm{~m})$ and an irregularly oscillating behaviour for $B L>35 \mathrm{~m}$. At first glance, this visibility function is reminiscent of that for a uniform disk, which is a Bessel function of the first kind of order 1: $\left|J_{1}(B L)\right|$. The direct comparison in Fig. A.1 reveals, however, significant discrepancies between such a Bessel function and the data. Additionally, our data shows no evidence for the $2 \pi$ phase jumps that occur at the zero crossings of the Bessel function. We thus conclude that a uniform disk model cannot explain the data.

We chose an alternative approach to analyse the data: first we interpolated $V_{\lambda}(B L)$ (blue curves in the top row of Fig. A.1) and then applied an inverse Fourier transform to this visibility function for each wavelength bin: $I_{\lambda}(d)=\mathcal{F}_{B L}\left[V_{\lambda}(B L)\right](d)$. This reconstruction method gives a first order estimate of the radial brightness distribution of the nucleus $I_{\lambda}(d)$, where $d$ is the angular distance from the centre. The shape of the visibility function $V_{\lambda}(B L)$ is only well determined for $20 \mathrm{~m}<B L<90 \mathrm{~m}$. This in turn implies that we have probed angular sizes $d$ between 10 and 70 mas, considering the entire wavelength range of the MIDI observations. These characteristic sizes in the Fourier and the real domain are indicated by vertical dashed and dotted lines in Fig. A.1.

The reconstructed brightness distribution is composed of two components plus a small fraction of extended flux at distances of more than 100 mas of the centre. The extended component is fully resolved by our interferometric set-up, while our measurements probe the properties of the other two components. One should bear in mind that we assumed axisymmetry for this analysis and already from the qualitative discussion of the data in Sect. 3 we know that this is a crude simplification. We therefore used the reconstructed brightness distribution only to extract the effective size of the emission region in the mid-infrared and traced its evolution depending on the wavelength. This was achieved by fitting a Gaussian distribution to the reconstructed radial profiles at every wavelength bin. This Gaussian is shown in green in the bottom row of Fig. A.1. The full width at half maximum (FWHM) of this Gaussian distribution is shown as a function of wavelength in Fig. A.2. We attribute the small dip in the size between 9.5 and $10.0 \mu \mathrm{m}$ to artefacts coming from the ozone feature (hatched area in Fig. A.2). From shorter to longer wavelengths, the size of the flux distribution increases slightly from 50 to 75 mas. This indicates that the emission at longer wavelengths is more extended than at short wavelengths: the temperature of the dust decreases with distance to the nucleus, but apparently only slowly.

These effective size estimates again demonstrate the need for interferometric observations, as they are the only way to reach these resolutions in the infrared.

\section{Appendix B: 2D geometrical model}

This second model is a two-dimensional, purely geometrical model, aimed to reconstruct the brightness distribution of the source from the full two-dimensional visibility data set. The model consists of two concentric, elliptical Gaussian distributions. It was motivated by evidence for two components in the one-dimensional analysis and by the dependence of the visibilities on the position angle which imply an elongated source (Sect. 3.2 and there especially Fig. 3). The brightness distribution has the following functional form ${ }^{4}$ :

$$
\begin{aligned}
& I(\lambda, \alpha, \delta)=f(\lambda) \cdot \exp \left(-4 \ln 2 \cdot\left[\left(\frac{\alpha \cos \phi(\lambda)+\delta \sin \phi(\lambda)}{r_{1}(\lambda) \cdot \Delta_{1}(\lambda)}\right)^{2}\right.\right. \\
& \left.\left.+\left(\frac{\alpha \sin \phi(\lambda)-\delta \cos \phi(\lambda)}{\Delta_{1}(\lambda)}\right)^{2}\right]\right) \\
& +\exp \left(-4 \ln 2 \cdot\left[\left(\frac{\alpha \cos \phi(\lambda)+\delta \sin \phi(\lambda)}{r_{2}(\lambda) \cdot \Delta_{2}(\lambda)}\right)^{2}\right.\right. \\
& \left.\left.+\left(\frac{\alpha \sin \phi(\lambda)-\delta \cos \phi(\lambda)}{\Delta_{2}(\lambda)}\right)^{2}\right]\right)
\end{aligned}
$$

Here, $\alpha$ and $\delta$ are the position on the sky with respect to the centre of the distribution. This two component model has a total of six free parameters: the sizes of the two Gaussians, given by their full width at half maximum $\Delta_{1}(\lambda)$ and $\Delta_{2}(\lambda)$; their oblateness, represented by the axis ratio $r_{1}(\lambda)$ and $r_{2}(\lambda)$; the position angle $\phi(\lambda)$ of the major axis with respect to north assumed to be the same for both components; and the relative intensity of the two components quantified $f(\lambda)$.

These parameters were then optimised using the following prescription: for a given wavelength $\lambda$ the flux distribution $I_{\lambda}(\alpha, \delta)=I(\lambda, \alpha, \delta)$ was generated according to the set of parameters. Then the Fourier transform $\mathcal{V}_{\lambda}(u, v)=\mathcal{F}_{\alpha, \delta}\left[I_{\lambda}(\alpha, \delta)\right](u, v)$ was calculated using a fast Fourier transform (FFT). The result corresponds to the visibility distribution in the $u v$ plane. The visibility values for the model were extracted from this plane at the positions $u_{i}=B L_{i} \cdot \sin \mathrm{PA}_{i}, v_{i}=B L_{i} \cdot \cos \mathrm{PA}_{i}$, using the baseline information from our observations $i$ consisting of the baseline length $B L_{i}$ and the position angle $\mathrm{PA}_{i}$. Finally, the modelled visibilities $V_{i}^{\bmod }(\lambda)=\left|\mathcal{V}_{\lambda}\left(u_{i}, v_{i}\right)\right|$ were compared to the observed ones $V_{i}^{\mathrm{obs}}(\lambda)$. The discrepancy was weighted with the measurement errors $\sigma_{V_{i}^{\text {obs }}}(\lambda)$ to obtain the $\chi^{2}$ :

$\chi^{2}(\lambda)=\sum_{i=1}^{21}\left(\frac{V_{i}^{\mathrm{obs}}(\lambda)-V_{i}^{\mathrm{mod}}(\lambda)}{\sigma_{V_{i}^{\mathrm{obs}}}(\lambda)}\right)^{2}$.

This $\chi^{2}$ was minimised using the Levenberg-Marquardt leastsquares minimisation algorithm. This process was performed independently for the 51 wavelength bins measured by MIDI between 8 and $13 \mu \mathrm{m}$. A comparison of the model to the data is shown for 8.5 and $12.5 \mu \mathrm{m}$ in Fig. B.1. This plot is the same as Fig. 4, except that here the visibilities are compared instead of the correlated fluxes. The resulting parameter dependencies are shown in Fig. B.2. To have more practical parameters, the factor $f$ was replaced by the ratio $R$ of the flux in the compact component to the flux in the extended component. The reduced $\chi^{2}$ (that is $\chi^{2}(\lambda) / N_{\text {free }}$ with $N_{\text {free }}=21-6=15$ degrees of freedom), shown in the left panel of Fig. B.3, varies strongly for different wavelengths. The fits are best in the middle of the wavelength range. Towards the edges of the $N$ band, the fits get significantly

${ }^{4}$ To be more descriptive, we use the FWHM, $\Delta_{n}=2 \sqrt{2 \ln 2} \sigma_{n}$, in our definition of the Gaussian distribution instead of the sigma, $\sigma_{n}$, in the standard definition. 
K. R. W. Tristram et al.: The nuclear dust torus in the Circinus galaxy, Online Material p 4
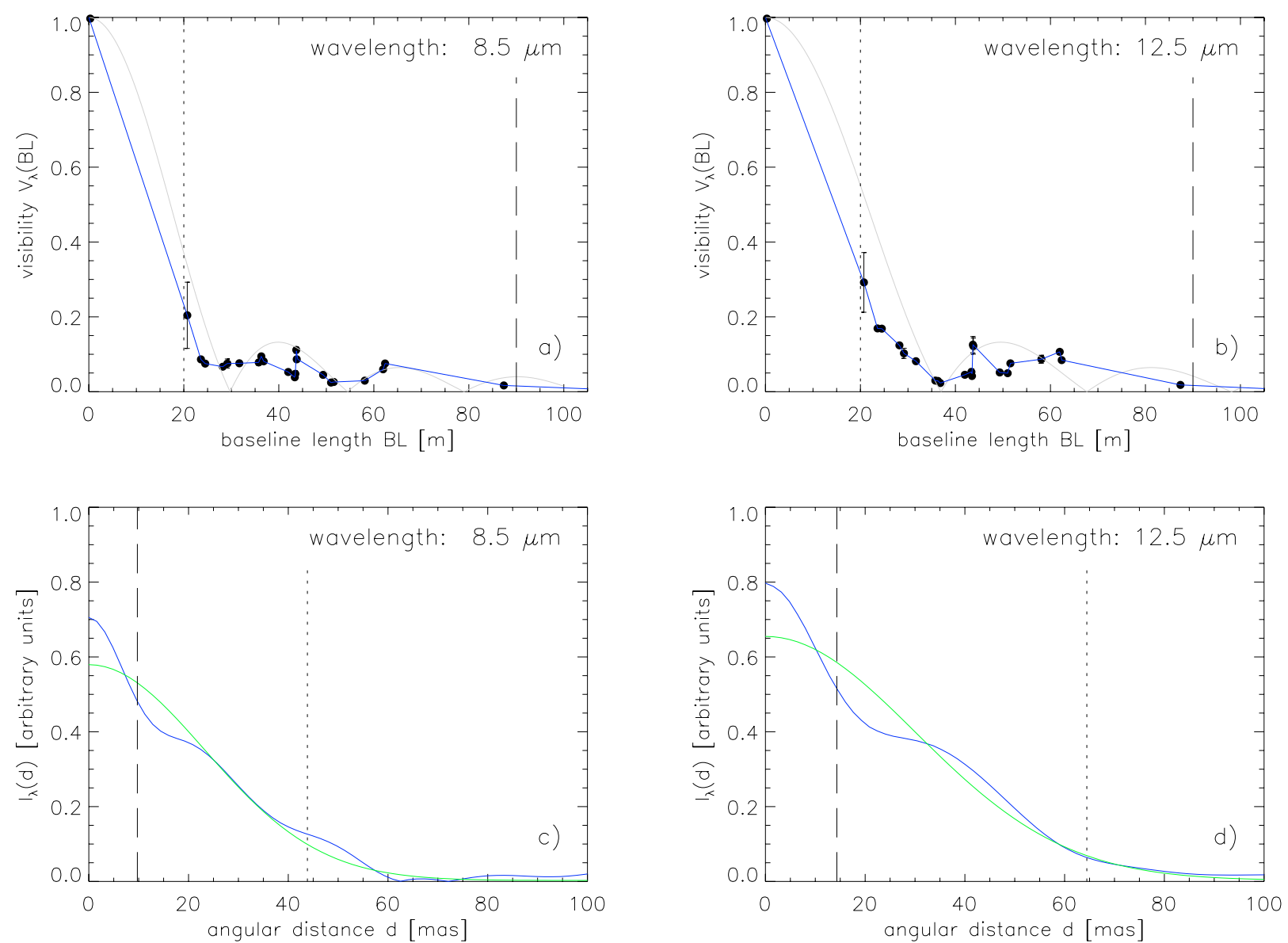

Fig. A.1. Radial 1D analysis of the mid-infrared emission in Circinus. Top row: visibility as a function of baseline length for two wavelength bins at a) $8.5 \mu \mathrm{m}$ and b) $12.5 \mu \mathrm{m}$ taking together all position angles. The data cannot be explained by a uniform disk model (light grey curves). The blue curve is an interpolated visibility function derived from the data. Bottom row, c) and d): For the same wavelengths, the inverse Fourier transform of the blue curve in the top row graphs is plotted, corresponding to a brightness distribution. Traced in green is the Gaussian distribution fitted to the Fourier transform in order to get an estimate for the size of the emitting source. The vertical lines indicate the angular size range probed by the interferometric measurements.

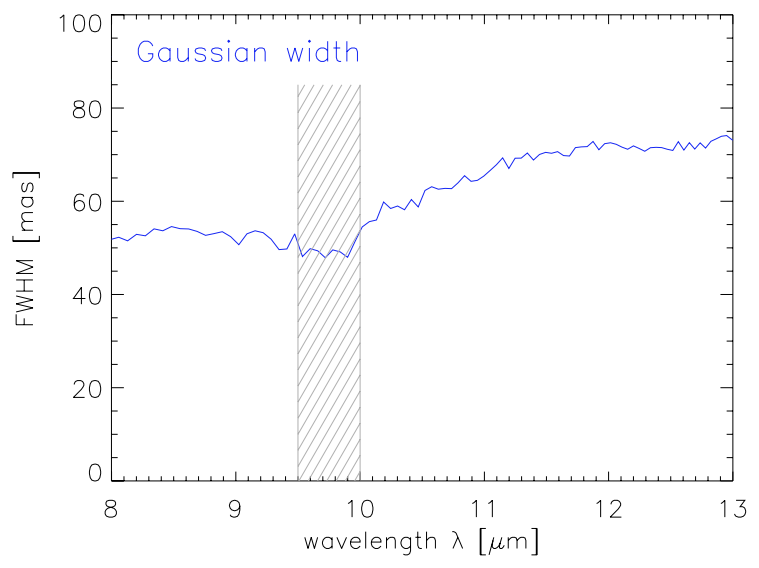

Fig. A.2. Characteristic size of the central dust emission in Circinus as a function of wavelength: the figure shows the $F W H M$ of the Gaussian distribution fitted to the radial profile of the brightness distribution of the Circinus nucleus (see Fig. A.1). This first estimate for the effective size of the nuclear dust emission shows a slow increase with wavelength from 50 to 70 mas. The area affected by atmospheric absorption are hatched in grey.

worse. This is mainly due to the weighting of the discrepancy between model and data using the errors of the data $\sigma_{i}^{\text {obs }}(\lambda)$. Due to the bad signal-to-noise ratio for low fluxes, the errors of the visibility are of the same order, or even larger within the silicate absorption feature, the atmospheric ozone feature, as well as at the edges of the $N$ band. The larger errors lead to a lower $\chi^{2}$ value. When considering a modified estimate for the quality of the fit,

$\xi^{2}(\lambda)=\sum_{i=1}^{21}\left(\frac{V_{i}^{\mathrm{obs}}(\lambda)-V_{i}^{\bmod }(\lambda)}{V_{i}^{\bmod }(\lambda)}\right)^{2}$,

the discrepancy is of the same order, except at $\lambda>12 \mu \mathrm{m}$. The reduced $\xi^{2}, \xi^{2}(\lambda) / N_{\text {free }}$, is shown in the right panel of Fig. B.3.

With the high values of the reduced $\chi^{2}$, the fit is comparatively bad. This is partly due to an overly optimistic estimate of the errors of the visibilities. The errors only represent the relative errors within one measurement and do not reflect the systematic errors between two measurements at different epochs. More importantly, we see in the bad fit quality evidence for a more complex structure of the source. This will be discussed in Sect. 4.2 .

Although no clear dependency of the parameters with wavelength is identifiable, a few general trends are present. The smaller component has a rather constant size of approximately $\Delta_{1} \sim 20$ mas and a rather high axis ratio of $r_{1}=0.2$. The position angle has a preferred orientation of $\mathrm{PA} \sim 40^{\circ}$, which 
K. R. W. Tristram et al.: The nuclear dust torus in the Circinus galaxy, Online Material p 5
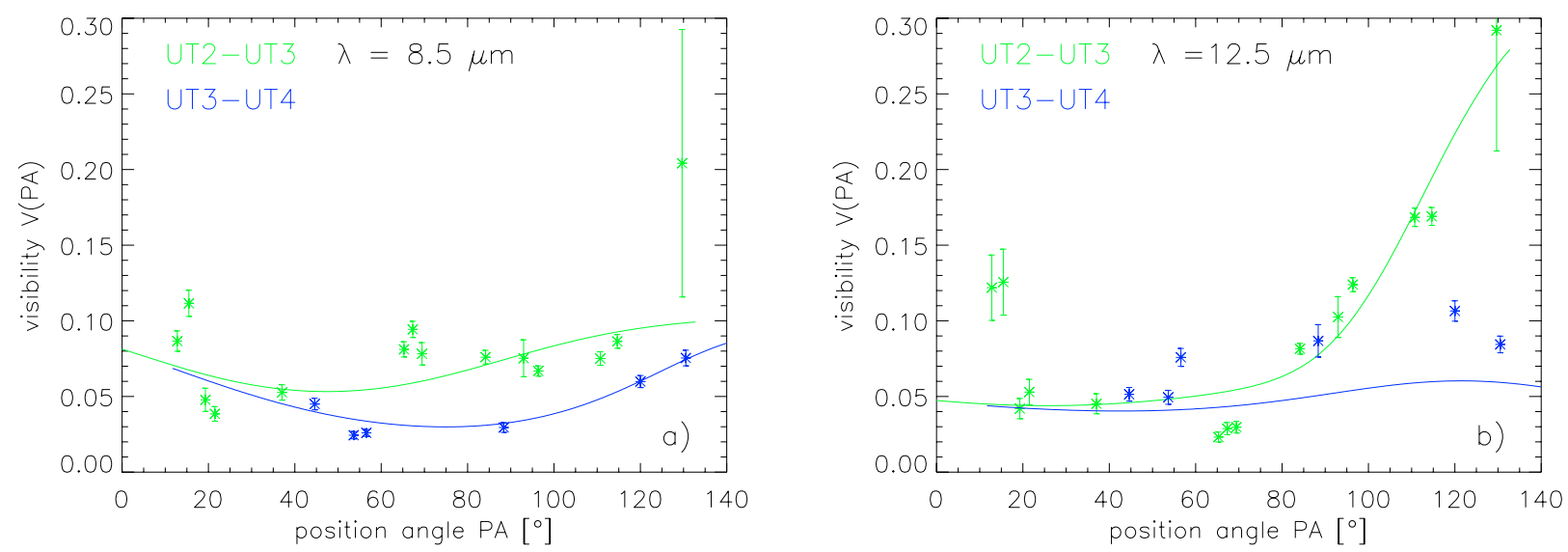

Fig. B.1. Comparison of the visibilities between the 2D geometrical model (two elliptical Gaussian distributions) and the measured data for two wavelengths: a) 8.5 and b) $12.5 \mu \mathrm{m}$. The data are plotted with asterisks; the modelled visibilities are the continuous lines. The two baselines shown here are plotted in two different colours, UT2 - UT3 in green (grey) and UT3 - UT4 in blue (black).
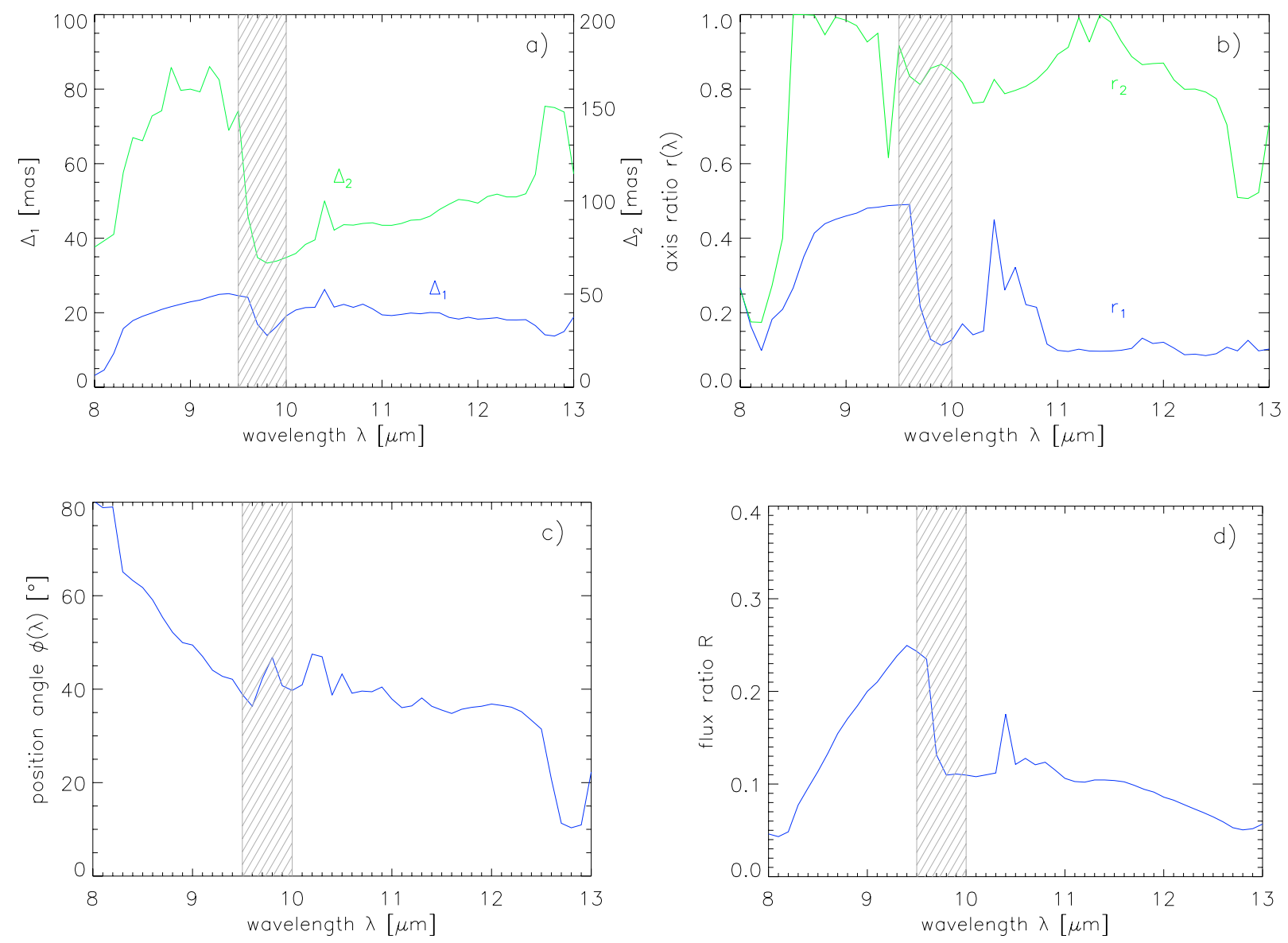

Fig. B.2. Wavelength dependence of the parameter estimates for the two-dimensional geometric model. The six parameters are a) the two $F W H M$ of the Gaussian components, b) the respective axis ratios $r, \mathbf{c}$ ) the position angle $\phi$ of the elliptical distribution and d) the ratio $R$ of the flux in the compact over the extended component. The areas affected by atmospheric absorption are hatched in grey.

is more or less perpendicular to the ionisation cone. Towards both ends of the spectral range sampled $(8.0 \mu \mathrm{m}<\lambda<9.0 \mu \mathrm{m}$ and $12.5 \mu \mathrm{m}<\lambda<13.0 \mu \mathrm{m})$, the position angle deviates significantly from this value. A more regular behaviour of the parameters is found in the range from 11.0 to $12.5 \mu \mathrm{m}$. Here also the intensity ratio stabilises at $R \sim 0.1$ and the size of the second component grows linearly with increasing wavelength. The large component hence comprises the bulk of the emission. The ellipticity of the larger component is relatively small, with $r_{2} \sim 0.75$ for large parts of the spectral range, again exceeding these margins at the edges of the spectrum sampled. Some of the parameters show a certain reciprocal influence: decreasing the inclination of the smaller component between 8.2 and $9.5 \mu \mathrm{m}$ leads to a larger emitting region and hence to a larger flux, which has an influence on the flux ratio between the two components and the size of the larger component. We hence do not see the decrease of ellipticity of the small component to be significant but rather as an artifact of the ambiguity of the data. Especially 
K. R. W. Tristram et al.: The nuclear dust torus in the Circinus galaxy, Online Material p 6
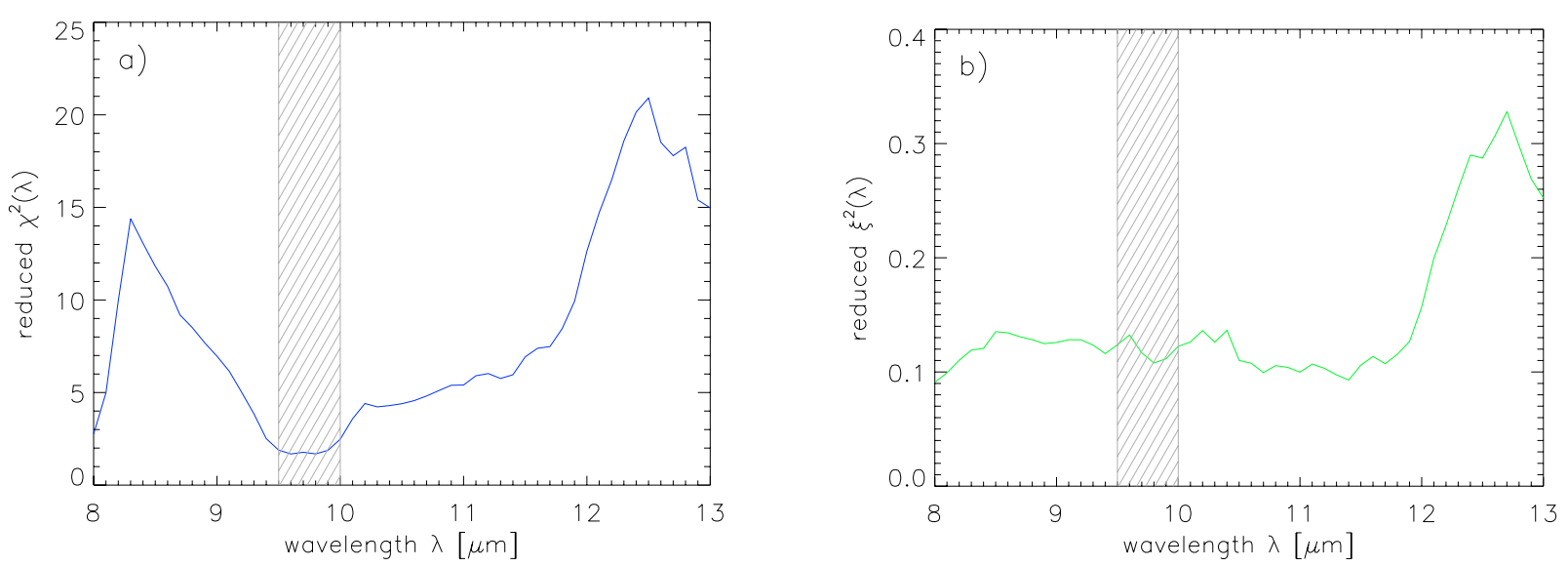

Fig. B.3. a) $\chi^{2}$ and b) $\xi^{2}$ for the fit of the two component Gaussian model to the visibilities. The areas hatched in grey are affected by atmospheric absorption.

the ellipticity, the size and the flux ratio of the two components show a certain degree of degeneracy. We found that, by fixing some of the parameters to average values (e.g. PA to $40^{\circ}$ or $r_{1}$ to 0.2 ), the quality of the fit for the individual wavelengths does not decrease significantly. At the same time, however, the behaviour of the other parameters, which are then adjusted, looks steadier. We did not follow this path of analysis any further but decided to apply a physical model instead (see Sect. 4.2). The overall erratic behaviour of the parameters and the quality of the fit, however, show that two Gaussians are not a good fit and that there are substantial differences at different wavelength bins. Nonetheless, we find that other models of the same simplicity produce no better fits. A more detailed discussion of alternative models is be given in Sect. 4.1. 
K. R. W. Tristram et al.: The nuclear dust torus in the Circinus galaxy, Online Material $p 7$

\section{Appendix C: Comparison of the full data set to the physical model}

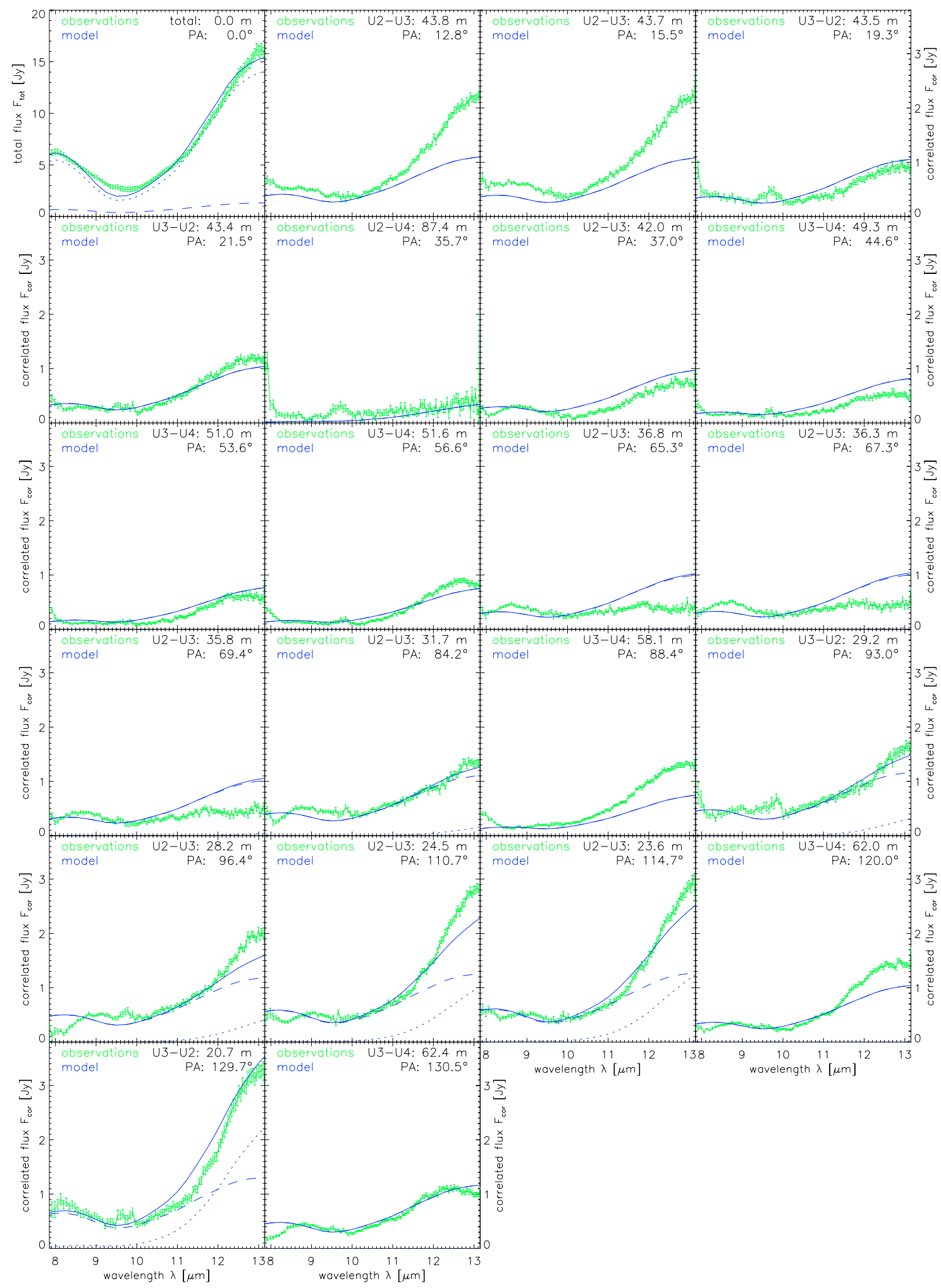

Fig. C.1. Comparison of the dispersed correlated fluxes (green/grey) to the model fluxes for the physical model fit (fit2, blue/black) in the entire wavelength range from 8 to $13 \mu \mathrm{m}$. The dashed line represents the flux contribution of the small disk component, while the dotted line is the contribution of the large torus component. 